\title{
Swarm robotics: a review from the swarm engineering perspective
}

\author{
Manuele Brambilla • Eliseo Ferrante • Mauro Birattari • \\ Marco Dorigo
}

Received: 31 May 2012 / Accepted: 29 November 2012 / Published online: 17 January 2013

(C) Springer Science+Business Media New York 2013

\begin{abstract}
Swarm robotics is an approach to collective robotics that takes inspiration from the self-organized behaviors of social animals. Through simple rules and local interactions, swarm robotics aims at designing robust, scalable, and flexible collective behaviors for the coordination of large numbers of robots. In this paper, we analyze the literature from the point of view of swarm engineering: we focus mainly on ideas and concepts that contribute to the advancement of swarm robotics as an engineering field and that could be relevant to tackle real-world applications. Swarm engineering is an emerging discipline that aims at defining systematic and well founded procedures for modeling, designing, realizing, verifying, validating, operating, and maintaining a swarm robotics system. We propose two taxonomies: in the first taxonomy, we classify works that deal with design and analysis methods; in the second taxonomy, we classify works according to the collective behavior studied. We conclude with a discussion of the current limits of swarm robotics as an engineering discipline and with suggestions for future research directions.
\end{abstract}

Keywords Swarm robotics $\cdot$ Review $\cdot$ Swarm engineering

\section{Introduction}

Swarm robotics has been defined as "a novel approach to the coordination of large numbers of robots" and as "the study of how large numbers of relatively simple physically embod-

Guest editor: Lynne E. Parker.

M. Brambilla $(\bowtie) \cdot$ E. Ferrante · M. Birattari · M. Dorigo

IRIDIA, CoDE, Université Libre de Bruxelles, 50 Av. Franklin Roosevelt, CP 194/6, 1050 Brussels,

Belgium

e-mail: mbrambilla@iridia.ulb.ac.be

E. Ferrante

e-mail: eferrante@iridia.ulb.ac.be

M. Birattari

e-mail: mbiro@ulb.ac.be

M. Dorigo

e-mail: mdorigo@ulb.ac.be 
ied agents can be designed such that a desired collective behavior emerges from the local interactions among agents and between the agents and the environment." (Şahin 2005).

The main characteristics of a swarm robotics system are the following:

- robots are autonomous;

- robots are situated in the environment and can act to modify it;

- robots' sensing and communication capabilities are local;

- robots do not have access to centralized control and/or to global knowledge;

- robots cooperate to tackle a given task.

In this review, we use these characteristics to discriminate the works that belong to swarm robotics from those that belong to other multi-robot approaches (Iocchi et al. 2001). Slightly different characterizations of swarm robotics have been proposed and adopted by Şahin (2005), Beni (2005) and Dorigo and Şahin (2004).

The main inspiration for swarm robotics comes from the observation of social animals. Ants, bees, birds, and fish are some examples of how simple individuals can become successful when they gather in groups. The interest towards social animals stems from the fact that they exhibit a sort of swarm intelligence (Bonabeau et al. 1999; Dorigo and Birattari 2007). In particular, the behavior of groups of social animals appear to be robust, scalable, and flexible.

Robustness is the ability to cope with the loss of individuals. In social animals, robustness is promoted by redundancy and the absence of a leader. Scalability is the ability to perform well with different group sizes. The introduction or removal of individuals does not result in a drastic change in the performance of a swarm. In social animals, scalability is promoted by local sensing and communication. Flexibility is the ability to cope with a broad spectrum of different environments and tasks. In social animals, flexibility is promoted by redundancy, simplicity of the behaviors and mechanisms such as task allocation. A detailed analysis of robustness, scalability, and flexibility in social animals has been carried out by Camazine et al. (2001).

By taking inspiration from social animals, swarm robotics aims at developing robotics systems that exhibit swarm intelligence features similar to those that characterize social animals. In particular, swarm robotics systems are meant to be robust, scalable, and flexible.

\subsection{Swarm engineering}

Swarm engineering is the systematic application of scientific and technical knowledge to model and specify requirements, design, realize, verify, validate, operate, and maintain a swarm intelligence system. Swarm engineering as a term was introduced by Kazadi (2000), who recognized that the focus of swarm intelligence research is moving towards "the design of predictable, controllable swarms with well-defined global goals and provable minimal conditions". He also adds that "to the swarm engineer, the important points in the design of a swarm are that the swarm will do precisely what it is designed to do, and that it will do so reliably and on time" (Kazadi 2000). However, the first work to formally introduce swarm engineering was published only five years later, with the seminal paper by Winfield et al. (2004).

Swarm engineering is still in a very early stage and its development is not homogeneous. On the one hand, some topics, such as design and analysis, have already received attention from the swarm robotics community and several methodologies and tools have been proposed. For these topics, our goal is to present and classify the existing works. On the 

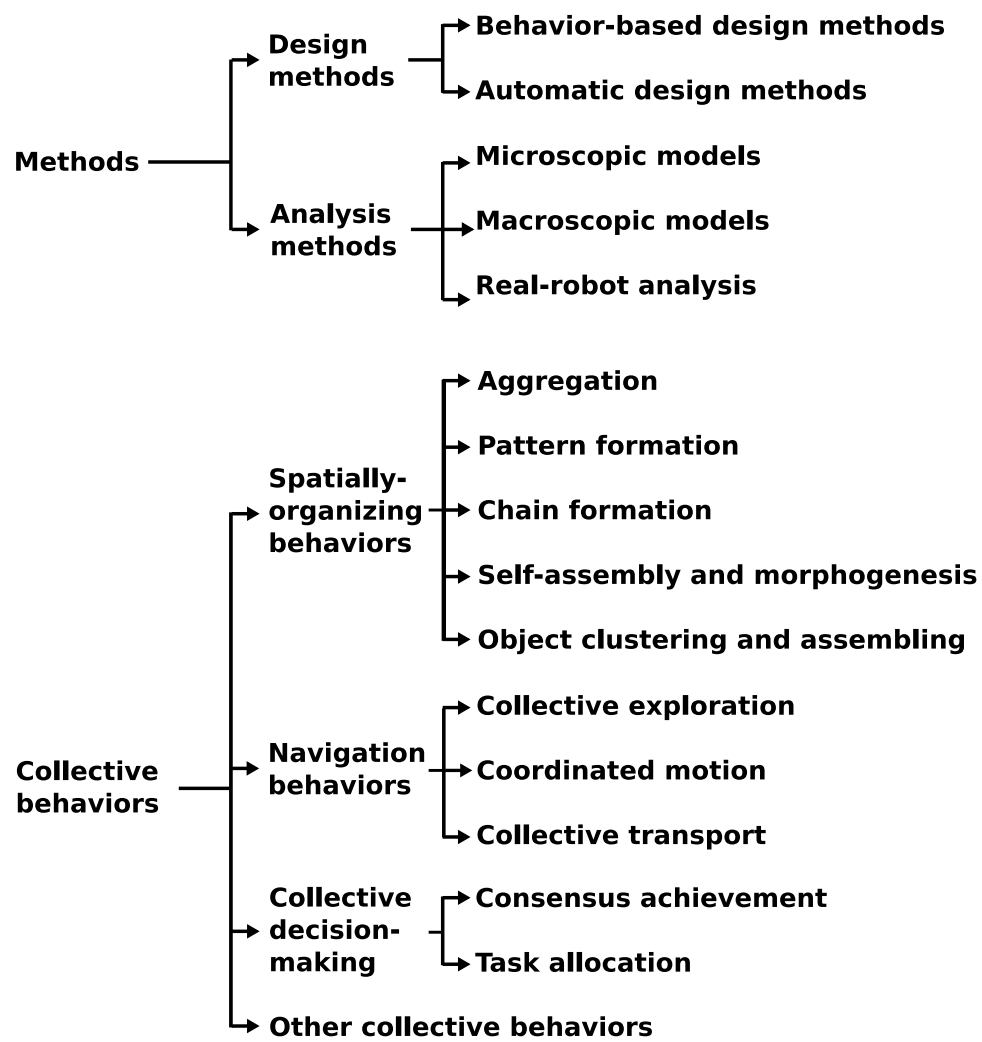

Fig. 1 The two taxonomies proposed in this review

other hand, other topics, such as requirements analysis, maintenance and performance measurement, have received almost no attention. In the last section of this review, we propose a discussion of these topics with the hope to foster new ideas and promote their development.

As we take a swarm engineering perspective, our review covers works that contributed to the advancement of swarm robotics as a field of engineering. In particular, we focus on ideas and solutions that promote the application of swarm robotics to real-world applications.

\subsection{The outline of our review}

In this review we use two taxonomies: methods and collective behaviors (see Fig. 1 for a full scheme of the structure of the review). In Sect. 2, we analyze methods to design and analyze swarm robotics systems. In Sect. 3, we analyze some of the possible collective behaviors a swarm robotics system can exhibit. By collective behaviors we mean behaviors of the swarm considered as a whole. Such collective behaviors can be used as building blocks for applications, such as foraging or construction (see also Sect. 4). In Sect. 4, we conclude the paper with a discussion of the open problems in swarm robotics and swarm engineering. 


\subsection{Previous reviews}

Previous reviews proposed taxonomies that differ from those that we propose here. Dudek et al. (1993) chose swarm size, communication range, communication topology, communication bandwidth, swarm reconfigurability and swarm unit processing ability to classify the literature. Cao et al. (1997) used: group architecture, resource conflicts, origins of cooperation, learning, and geometric problems. Iocchi et al. (2001) adopted a hierarchical taxonomy: in the first level they considered aware versus unaware cooperation. The aware category is divided into strongly coordinated, weakly coordinated and not-coordinated systems. Works related to strongly coordinated systems are divided into strongly centralized, weakly centralized and distributed. A separate section is dedicated to applications of multi-robot systems. Gazi and Fidan (2007) chose to divide the literature into mathematical models, swarm coordination and control, and design approaches. Bayindir and Şahin (2007) classified the literature according to five taxonomies: modeling, behavior design, communication, analytical studies and problems.

\section{Methods}

The goal of this section is to classify the articles published in the swarm robotics literature according to the methods used to design or to analyze swarm robotics systems. In Sect. 2.1, we present the most common design methods used to develop collective behaviors for swarms of robots. In Sect. 2.2, we present the most common methods used to understand, predict, and analyze the collective behavior of a swarm.

\subsection{Design methods}

Design is the phase in which a system is planned and developed starting from the initial specifications and requirements. Unfortunately, in swarm robotics there are still no formal or precise ways to design individual level behaviors that produce the desired collective behavior. The intuition of the human designer is still the main ingredient in the development of swarm robotics systems.

We divide the design methods into two categories: behavior-based design and automatic design.

Behavior-based design is the most common way to develop a swarm robotics system. In an iterative way, the individual behavior of each robot is implemented, studied, and improved until the desired collective behavior is obtained. In behavior-based design, inspiration is often taken from the observation of the behaviors of social animals. This may ease the design process as, sometimes, the details of a particular behavior are already understood and mathematical models are available.

Another way to develop swarm robotics systems is via automatic design methods. Automatic design methods can be used to reduce the effort of the developers in creating a collective behavior. We classify automatic design methods in two categories: evolutionary robotics and multi-robot reinforcement learning.

In the following, we discuss behavior-based design methods and automatic design methods by describing the general principles and the relative advantages and disadvantages. 
Fig. 2 The response threshold function. The transition probability $p$ depends on: $s$, a stimulus that represents a measure of the transition urgency; $\theta$, a threshold on the stimulus; and $\beta$, a sensitivity parameter. The function is non-linear: When $s \ll \theta$, the transition probability is very low, whereas when $s \gg \theta$ it is very high. In the example in the figure, $s$ ranges in $[0,100], \theta=50$ and $\beta=8$

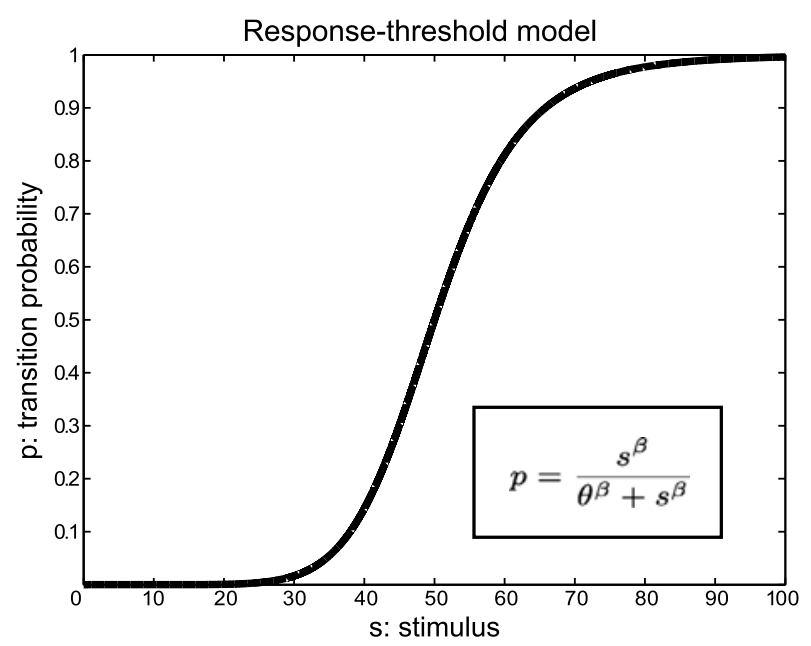

\subsubsection{Behavior-based design methods}

In swarm robotics, the most commonly used design method involve developing, by hand, the individual behaviors of the robots which results in the collective behavior of the swarm. Designing a behavior for a swarm robotics system is usually a trial and error process: individual behaviors are iteratively adjusted and tuned until the resulting collective behavior is obtained. For this reason, behavior-based design is, typically, a bottom-up process (Crespi et al. 2008) even though some top-down methods have been recently proposed (see Sect. 2.1.1).

We divide the literature on behavior-based design methods into three main categories: probabilistic finite state machine design, virtual physics-based design and other design methods.

Probabilistic finite state machine design Generally, in swarm robotics, an individual robot does not plan its future actions, but it takes decisions only on the basis of its sensory inputs and/or its internal memory (Brooks 1986). One of the most adopted design methods to obtain such behaviors is the use of a particular class of finite state machine (Minsky 1967): probabilistic finite state machines (PFSMs).

In PFSMs, the transition probability between states can be fixed or can change over time. The transition probability is fixed when a single probability value is defined and used throughout the execution of the collective behavior. An example can be found in the work of Soysal and Şahin (2005). The transition probability is not fixed when it is defined through a mathematical function of one or more parameters of the system. One of the most widely used function is the response threshold function developed by Granovetter (1978) (see also Bonabeau et al. 1997). The response threshold function, depicted in Fig. 2, has been used to study the collective behavior of social insects (Theraulaz et al. 1998), and has been introduced in swarm robotics by Theraulaz et al. (1990) to study collective decision-making and task allocation. In the response threshold function, the probability to switch to a new state is usually related to the current state of the robot.

PFSMs have been used to develop several collective behaviors, such as aggregation (Soysal and Şahin 2005), chain formation (Nouyan et al. 2008) and task allocation (Liu et al. 2007; Labella et al. 2006). These behaviors will be explained in more detail in Sect. 3.1.1, Sect. 3.1.2 and Sect. 3.3.2, respectively. 
Fig. 3 The Lennard-Jones potential function. The potential $v$ depends on the current distance $d$ between two robots. $\sigma$ is the desired distance between the robots and $\epsilon$ corresponds to the depth of the potential function. In this example, $\sigma=0.3$ and $\epsilon=2.5$

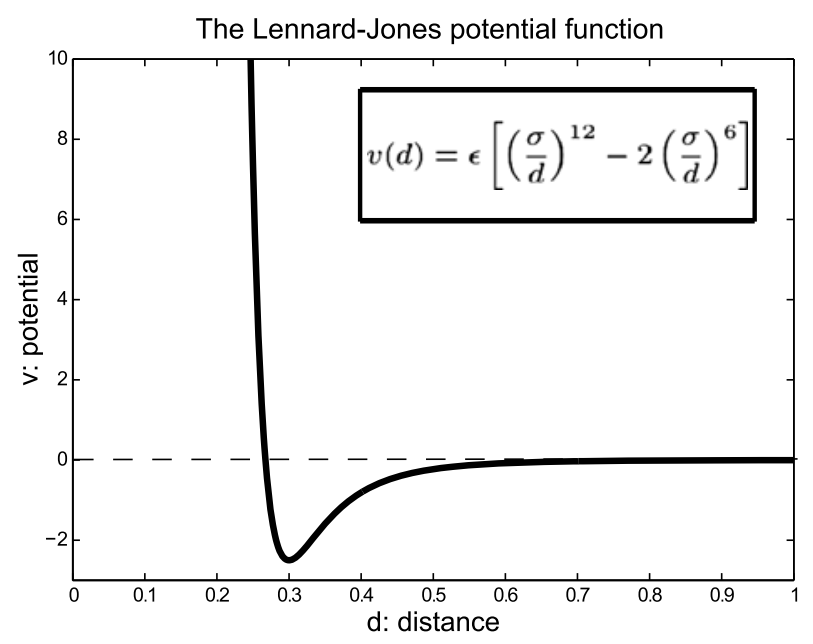

Virtual physics-based design The virtual physics-based design method draws inspiration from physics. Each robot is considered as a virtual particle that exerts virtual forces on other robots.

One of the first works using virtual physics-based design was by Khatib (1986), who used the concept of artificial potential field. In this and in some following works, the robots are subject to repulsive virtual forces originating from the environment: the goal is associated with an attractive force and the obstacles with repulsive forces. The social potential fields framework (Reif and Wang 1999) considers also robots as associated to virtual forces. Later, Spears et al. (2004) proposed a virtual physics-based design method called physicomimetics framework. Since we believe this is the most general framework, it will be used to describe the method. In describing virtual physics-based design, we will follow the most common used terminology, which uses, sometimes in an inaccurate way, a vocabulary borrowed from physics.

Virtual physics-based design assumes that the robots are able to perceive and distinguish neighboring robots and obstacles, and to estimate their distance and relative position. Each robot computes a virtual force vector $\mathbf{f}=\sum_{i=1}^{k} f_{i}\left(d_{i}\right) e^{j \theta_{i}}$, where $\theta_{i}$ and $d_{i}$ are the direction and the distance of the $i$ th perceived obstacle or robot and the function $f_{i}\left(d_{i}\right)$ is derived from an artificial potential function. The most commonly used artificial potential is the LennardJones potential, depicted in Fig. 3.

The main advantages of virtual physics-based design methods are: (i) a single mathematical rule smoothly translates the entire sensory inputs space into the actuators output space without the need for multiple rules or behaviors; (ii) the obtained behaviors can be combined using vectorial operations; (iii) some properties (such as robustness, stability, etc.) can be proved using theoretical tools from physics, control theory or graph theory (Gazi and Passino 2002).

The virtual physics-based method is often used to design collective behaviors that require a robot formation. Examples of such behaviors are pattern formation (Sect. 3.1.2), collective exploration (Sect. 3.2.1) and coordinated motion (Sect. 3.2.2).

Other behavior-based design methods In this section, we outline other works using behavior-based design that do not fit in the previous sections.

Bachrach et al. (2010) proposed a scripting language called Protoswarm based on the amorphous computational medium (Beal 2004). The amorphous computational medium 
considers the environment as filled with individuals able both to perform computations and to communicate with their neighbors. Using Protoswarm it is possible to define behaviors for an individual by writing scripts at the collective level. Several collective-level primitives exist in the scripting language, both related to space and time. These primitives are translated into individual behaviors by exploiting the underlying local communication. This language, even though it cannot be considered a standalone design method, can significantly ease the design process thanks to its collective-level primitives. This approach is particularly suited for sensor networks, where the great number of individuals guarantees that the system is able to cover the entire environment with a single communication network.

Brambilla et al. (2012) proposed a top-down method to design swarm robotics systems. Their idea is to define the desired system using a set of properties. These properties are logic formulas which need to hold true in the final system. The authors propose an iterative process composed of four steps. In the first step, the properties are defined. In the second step, a macroscopic model is produced. A model checker is then used to verify that the properties hold true in the produced model. In the third step, the macroscopic model is used to guide the process of implementing the system using a simulator. Finally, in the fourth the system is tested using real robots. This approach can guide the design process from the desired system to the actual implementation. Moreover, the process helps to formally verify that the final system satisfies the desired properties.

\subsubsection{Automatic design methods}

The use of automatic design methods allows the automatic generation of behaviors without the explicit intervention of the developer.

Automatic design methods for swarm robotics systems can be divided in two main subdomains: reinforcement learning and evolutionary robotics. Panait and Luke (2005) conducted an extensive review of the state of the art of automatic design methods for multiagent and multi-robot systems. Differently from Panait and Luke (2005), in this review we focus on understanding the obtained results and the challenges of applying automatic design methods in swarm robotics.

The section is organized as follows. We first introduce reinforcement learning (Kaelbling et al. 1996; Sutton and Barto 1998) and we identify the key challenges in the application of the methods developed for reinforcement learning to swarm robotics. We then present evolutionary robotics (Nolfi and Floreano 2000), the application of evolutionary computation techniques to single and multi-robot systems. Finally, we present some individual works on automatic design methods that do not belong to either of the above two categories.

Reinforcement learning A set of methods to automatically design individual behaviors for robots in a swarm can be found in the reinforcement learning (RL) literature. RL traditionally refers to a class of learning problems: an agent learns a behavior through trial-and-error interactions with an environment and by receiving positive and negative feedback for its actions. In this section, we do not go into the details of RL: our goal is to discuss to what extent the methods developed for RL are or can be applied to swarm robotics. For a more formal introduction and more details about RL, the interested reader can refer to Kaelbling et al. (1996).

In RL, the robot receives a reward for its actions. The goal of the robot is to learn automatically an optimal policy, that is, the optimal behavior mapping robot states to robot actions. The behavior is optimal in the sense that it maximizes the rewards received from the environment. 
RL has been intensively studied in the single robot case where an elegant and unified mathematical framework has been developed (Kaelbling et al. 1996; Sutton and Barto 1998). In the multi-robot case, only few works with limited scope exist. A review of such works was conducted by Panait and Luke (2005), Yang and Gu (2005), and Stone and Veloso (2000).

A swarm robotics problem can hardly be seen as a RL problem. In fact, the swarm engineer tackles the task at the collective level, but learning typically takes place at the individual level. Thus, in applying methods developed for RL to swarm robotics, the main issue is the decomposition of the global reward into individual rewards (Wolpert and Tumer 1999). This challenging problem is called spatial credit assignment. Matarić $(1997,1998)$ addressed this issue by performing experiments with few robots ( 2 to 4 ), using communication or signaling to share the reward (Matarić 1997, 1998).

In addition to the spatial credit assignment, there are also other open problems: (i) The size of the state space faced in RL problems is huge. The reason behind this problem is the high complexity of the robots hardware and the complexity of the robot-to-robot interactions. Examples of techniques to reduce the state space dimension have been used by Riedmiller et al. (2009). In this work, the authors applied neural networks as function approximators together with fast learning algorithms (Kalyanakrishnan and Stone 2007). (ii) The environment perception is incomplete. This makes the search of the behavior even more complex (Kaelbling et al. 1998). Matarić and her colleagues addressed this problem using communication (Matarić 1998) or behavioral decomposition (Matarić 1997). (iii) The environment, as seen from the individual robot perspective, is non-stationary due to the fact that each robot's action is influenced by the actions performed by other robots in the same environment or by changes in the environment itself. We do not know of any work in multirobot learning addressing this problem.

Evolutionary robotics Evolutionary robotics (Nolfi and Floreano 2000) (henceforth ER) is an automatic design method that applies evolutionary computation techniques (Goldberg 1989; Holland 1975) to single and multi-robot systems. Evolutionary computation is inspired by the Darwinian principle of natural selection and evolution. As such, it uses a vocabulary borrowed from biology.

Within swarm robotics, ER has been used in many proof-of-concept tasks in order to test the effectiveness of the method (Baldassarre et al. 2007; Groß and Dorigo 2008a; Sperati et al. 2008) or as a tool to answer some more fundamental scientific questions (Trianni and Dorigo 2006; Tuci et al. 2004; Pini and Tuci 2008; Ampatzis et al. 2008). In this review, we analyze ER from an engineering perspective, that is, we describe its strengths and weaknesses as a design method.

The ER method can be described by the following process. At the beginning, a population of individual behaviors is generated at random. In each iteration, a number of experiments for each individual behavior is executed. The same individual behavior is used by all the robots in the experiment. In each experiment, a fitness function is used to evaluate the collective behavior of the swarm resulting from that individual behavior. At this point, a selection of the highest scoring individual behaviors are modified by genetic operators, such as cross-over and mutation, and used for the subsequent iterations. In the majority of the works, the group is homogeneous (all individual behaviors are the same) and the fitness evaluates the performance of the entire swarm. Waibel et al. (2009) introduced two taxonomies: one of the taxonomies distinguishes works according to how fitness is computed (individual-level vs swarm level), whereas the other one according to how the swarm is composed (homogeneous vs heterogeneous).

In ER, the individual behavior can be represented in many ways, such as finite state machines or virtual force functions (Hettiarachchi 2007). Typically, the evolutionary method 
is used to find the parameters of an artificial neural network (henceforth NN). Although several types of $\mathrm{NN}$ exist in the literature, they can roughly be categorized in two main classes: feed-forward NN (Fine 1999) and recurrent NN (Beer and Gallagher 1992; Elman 1990). Feed-forward NNs are used for individual behaviors that require no memory of previous observations and actions. Conversely, recurrent NNs are used for individual behaviors that require a memory of previously seen input patterns. ER with recurrent neural networks has been extensively studied in swarm robotics by Ampatzis (2008).

RL and ER have many common points. In fact, the same problems identified by us in the application of RL to swarm robotics apply also to ER. Other problems, many of which were identified by Matarić and Cliff (1996), are instead related only to ER: (i) Evolution is a computationally intensive process that does not give any guarantees on its convergence to a solution; (ii) neural networks are black-box and it is often very difficult to understand their behavior; (iii) from an engineering point of view, the complexity of behaviors currently synthesized through artificial evolution is relatively low and the same results may often be achieved by designing the behavior by hand.

Other learning and automatic design methods In this section, we outline other works on automatic design that can be placed neither within the RL nor within the ER literature. In all these works, the authors design an individual behavior with parameters to be found, and they use some algorithm to automatically find (or learn) these parameters on-line, that is, while the robots are interacting with the environment.

Parker (1996) proposed ALLIANCE, a multi-robot architecture that focuses on the achievement of fault tolerant, robust, and adaptive task allocation in a team of robots. In her work, the author added the possibility to perform on-line learning of the parameters of ALLIANCE.

Lee and Arkin (2003) extended their learning momentum framework to multi-robot systems. With learning momentum, it is possible to learn on-line the behavioral parameters according to the situations robots are facing. These parameters are the weight assigned to vectors produced by different, virtual physics-based, sub-behaviors.

Li et al. (2004) proposed an algorithm that enables on-line learning of some parameters of the robot behaviors in order to achieve diversity and specialization in a swarm of robots. The learning algorithm is specifically thought for their application, which consists of a stick pulling task.

Hettiarachchi (2007) used the virtual-physics-based design method combined with evolutionary computation. He used genetic algorithms to learn off-line the parameters of the Lennard-Jones potential function (see Sect. 2.1.1) in a navigation with obstacle avoidance task.

Rosenfeld et al. (2008) studied the problem of how to obtain an adaptive coordination behavior in a multi-robot domain. Their first contribution has been to propose a method that enables each robot to estimate the coordination cost over time. An example of such cost is the one needed to reduce interference. They then proposed a learning algorithm that is able to produce a behavior mapping the current estimate of the coordination cost to the coordination method to be used.

Finally, Pugh and Martinoli (2007) compared the PSO (particle swarm optimization) algorithm against a genetic algorithm for on-line learning parameters for a swarm of robots performing obstacle avoidance. They also defined metrics to measure diversity and specialization, and concluded that PSO is able to achieve a higher degree of diversity in the swarm. 


\subsection{Analysis}

Analysis is an essential phase in an engineering process. In the analysis phase, the swarm engineer is interested in seeing whether a general property of the designed collective behavior holds or not. The ultimate goal to obtain is that a swarm of real robots exhibits the desired collective behavior with the desired properties. Properties of the collective behaviors are usually analyzed by means of models.

Swarm robotics systems can be modeled at two different levels: the individual level, or microscopic level, which models the characteristics of the single individuals and the interactions among them; the collective level, or macroscopic level, which models the characteristics of the entire swarm. The development of models for analyzing swarm robotics systems at both levels of abstraction is still a subject of study and research. In fact, modeling both the microscopic and the macroscopic level and their interaction is very difficult due to the nature of self-organized systems (Abbott 2006). As a consequence, the vast majority of modeling techniques that are used nowadays focus on one level at a time.

In this review, we classify the literature on modeling according to whether the main concern is to capture the microscopic (Sect. 2.2.1) or the macroscopic (Sect. 2.2.2) aspects. In the last section (Sect. 2.2.3), we conclude with an overview of how the analysis with real robots is conducted.

\subsubsection{Microscopic models}

Microscopic models take into account each robot individually, analyzing both robot-to-robot and robot-to-environment interactions. The level of detail considered in microscopic models can vary greatly and influences the model and the results that can be obtained.

In the swarm robotics field, many models have been developed with different levels of abstraction: the simplest models consider the robots as point-masses; intermediate complexity models consider 2D worlds with kinematic physics; more complex models consider 3D worlds with dynamic physics where the details of each sensor and actuator are modeled. For an analysis of the different levels of abstraction see Friedmann (2010). In microscopic models, the behavior of each individual robot is also explicitly modeled. These individualbehavior models are mainly used for design purposes. As such they have already been presented in Sect. 2.1.1 of our review.

Microscopic models in which the elements composing a system are simulated with the use of a computer are traditionally called simulations. Simulations are among the most used tools to analyze and validate swarm robotics systems. The vast majority of the works presented in Sect. 3 have been analyzed using simulators. Simulators for swarm robotics systems have many characteristics in common with simulators for other mobile robotics systems. However, a unique feature of swarm robotics is the presence of a large number of robots. Unfortunately, scalability with respect to the number of robots is not the main concern for the vast majority of multi-robot simulators. Vaughan (2008) proposed a benchmark to study scalability in multi-robot simulators and applied it to the Stage simulator. Pinciroli et al. (2012) developed a simulator for swarm robotics by focusing explicitly on the scalability issue which was able to simulate $10^{5}$ robots in real time.

For a survey of various simulation platforms in robotics see Kramer and Scheutz (2007).

\subsubsection{Macroscopic models}

Macroscopic models consider swarm robotics systems as a whole. The individual elements of the systems are not taken into account in favor of a description of the system at a higher level. 
In this section we provide a broad overview of the main contributions in this area. We classify works in macroscopic modeling into three categories. In the first category, we consider works resorting to rate or differential equations. In the second category, we consider works where classical control and stability theory are used to prove properties of the swarm. In the third category, we consider other approaches.

Rate and differential equations One of the first works that uses rate equations for modeling swarm robotics systems is by Martinoli et al. (1999). In this and in follow-up works, the term rate equations was used to denote such models. Rate equations describe the time evolution of the proportion of robots in a particular state over the total number of robots.

Rate equations can be used to derive a macroscopic model of a collective behavior, starting from an individual-level PFSM. The procedure is the following: (i) First, a set of variables is defined. Usually, one variable is defined for each state of the individual-level PFSM. These variables are used to track the proportion of the robots in the corresponding states. (ii) Second, for each variable, an equation is defined (Lerman and Galstyan 2002). This equation is called rate equation because it is used to describe the time evolution of that variable, that is, the time evolution of the proportion of the robots in the corresponding state. The rate equation contains a set of parameters, one for each input and output transition of the corresponding state. Numerically, these parameters can be derived either from the description of the system or empirically.

The rate equations method was used to model many swarm robotics systems. In their seminal work, Martinoli et al. (1999) used rate equations to model a clustering task where robots gather objects. Lerman et al. (2001) and Martinoli et al. (2004) used rate equations to model the stick pulling experiment, a task where two robots need to cooperate in order to pull sticks out of their holes. Lerman and Galstyan (2002) modeled the foraging task under the effect of interference. In this case, the authors were correctly able to model the individual performance in foraging to be a decreasing function of group size. Trianni et al. (2002) used rate equations to model a chain formation behavior and an aggregation behavior, implemented using probabilistic finite state machines. Campo and Dorigo (2007) modeled the collective behavior of robots performing multi-foraging (i.e., foraging with more than one food source). Winfield et al. (2008) used rate equations to model a swarm of robots whose goal is to stay together while avoiding collisions. Liu and Winfield (2010) used the rate equations to model another foraging task involving the collection of energy units. Finally, O'Grady et al. (2009b) used rate equations to model an aggregation collective behavior. In this work, a flying robot can actively control the number of robots aggregating beneath it. The model included probabilities to join and to leave an aggregate, which were then directly used in the behavior design.

The main advantage of the rate equation approach is that it is a systematic method to translate microscopic models into macroscopic models. Its main limit is that, in general, it is difficult to model space and time: robots' positions in space are not explicitly modeled and discrete time is usually assumed; furthermore, the two mentioned limitations imply that each robot can change its position to any other location in the environment at each timestep. Galstyan et al. (2005) extended the rate equation model to include spatiality in a task where robots search for a chemical substance. However, the authors did not perform any experiments, simulated or with real robots, to validate their approach.

A recent advancement in macroscopic modeling based on differential equations is due to Hamann and Wörn (2008). Their models include noise, stochasticity, and spatiality. The basic building block of this modeling method are the Langevin equation and the FokkerPlanck equation, both borrowed from the statistical physics literature. 
The Langevin equation is a family of stochastic differential equations that describes the motion of a particle in a fluid. The Langevin equation is a mesoscopic model (intermediate level between micro and macro). In fact, the motion of the particle is modeled using two components: a deterministic component, which represents the microscopic laws of motion of that particle, and a stochastic component, which represents the interaction of the particle with the environment (in this case the ensemble of particles composing the fluid). In the case of a robot, the deterministic component of the Langevin equation models the deterministic motion of the robot influenced by its individual behavior, whereas the stochastic part models the interaction of the robot with the other robots (considered as a flow) and with the environment.

The Fokker-Plank equation can be used to describe the dynamics of the entire swarm. It models the time-evolution of the probability density function that describes the state (for example the position or the velocity) of all particles, or robots, in the environment. The derivation of the Fokker-Planck equation starting from the Langevin equation is possible using tools of statistical mechanics plus some problem-dependent intuition. Hamann and Wörn (2008) applied this modeling method to analyze coordinated motion (which they call collective taxis), aggregation (which they call collective perception) and foraging. Recently, the authors modeled aggregation in presence of a temperature gradient in the environment, and provided a comparison with another model called Stock \& Flow (Schmickl et al. 2009). The Fokker-Planck equation approach has the advantage that it can be used, in principle, to model any swarm robotics collective behavior. The two main disadvantages, however, are the following: the Fokker-Plank equation is difficult to be solved analytically and sometimes requires computationally demanding numerical algorithms; communication aspects, at present, are very difficult to model.

A similar approach was adopted also by Berman et al. (2009), who used a set of advection-diffusion-reaction partial differential equations to derive the individual behaviors of a swarm performing task allocation. In Berman et al. (2011b), this approach is applied to an area coverage task. Dantu et al. (2012) compared the results obtained in this work with those obtained from a simulation of the same behavior. The goal of the authors was to understand the effects of noise and errors on the collective behavior.

Another interesting study on the use of the Fokker-Planck equation was done by Prorok et al. (2011). In their work, the authors compared four different models of an area coverage behavior. They did it by measuring the area covered by the robots. Each model is characterized by being microscopic or macroscopic, and spatial or non-spatial. The predictions obtained from the models are compared with the results of both simulated and real-robot experiments. The authors showed that predictions of the spatial and non-spatial models differ for short time spans, for which the results of spatial models are more accurate, but are very similar for long time spans.

Classical control and stability theory The second set of works uses classical control and stability theory to prove properties of the swarm. Liu et al. (2003) and Gazi and Passino (2005) modeled a swarm of agents in a one-dimensional space using discrete-time discreteevent dynamical systems. Liu and Passino (2004) and Gazi and Passino (2004b) used Lyapunov stability theory to prove that the behavior studied was able to let a swarm achieve coherent social foraging in presence of noise. Similarly, Gazi and Passino (2003, 2004a) proved that, in specific conditions, a swarm of agents aggregates in one point of the environment. Schwager et al. (2011) modeled a swarm of communicating robots as a linear, discrete-time dynamical system. The authors then used their model to study how different communication topologies affect the Lyapunov stability of the system. Finally, Hsieh et al. 
(2008) used delay differential equations to model task allocation (agents allocating and reallocating to different physical sites), proving the stability of the reached configuration. In the same work, the authors also proposed a method to compute the optimal transition matrix in order to obtain a swarm that reaches the desired configuration.

All these modeling methods have the advantage to be based on strong mathematical formulations. However, the main problem with these methods is that they rely on many assumptions that, in swarm robotics, are often violated because of noise, asynchronicity, stochasticity, and absence of global information. Extensions of classical methods that tackle one of these elements individually do exist, but we are not aware of any work that tackles all these elements together.

Other modeling approaches In the third and final category we consider works in modeling that resort to other mathematical frameworks. Winfield et al. (2005) and Dixon et al. (2011) used linear time temporal logic to define properties of individual robots and of the swarm. The authors defined and proved two properties of the system: safety and liveness. The safety property is verified when the robots do not exhibit undesirable behaviors. The liveness property is verified when the swarm dynamics actually do evolve over time. A similar approach was used by Konur et al. (2012) and Brambilla et al. (2012). These authors analyzed swarm robotic systems by using probabilistic model checking, a technique that allows one to formally verify whether a given property is satisfied by a given model.

Massink et al. (2012) used Bio-PEPA to analyze consensus achievement in a robot swarm. Bio-PEPA is a high level modeling language. From a description of a system formulated in Bio-PEPA, one can automatically derive different models apt to perform stochastic simulation, fluid flow (ODE) analysis, and model checking. These models are guaranteed to be logically consistent.

Kazadi (2009) expressed the properties of a swarm using a mathematical language and proved their validity. The author proposed a way to define properties of a swarm robotics problem which he calls "model independent", that is, they do not depend on the actual implementation of the agent/robot. He proposed model-independent properties for two collective behaviors: shape formation and flocking. Soysal and Şahin (2007) modeled aggregation using Markov chains and validated the prediction using simulation.

The work of Turgut et al. (2008b) represents one of the first modeling attempts to bridge studies of flocking within physics with studies of flocking within robotics. In their study, the authors modeled alignment in flocking. The model shows that there is a phase transition from aligned to non-aligned state corresponding to a critical value of noise. The results were validated using simulation.

Correll (2008) used a population dynamics model to find the parameters used in two task-allocation behaviors. Using the model and an optimization algorithm, the authors could estimate the parameters that lead to the optimal distribution of robots.

Mathews et al. (2010) modeled a problem in which a flying robot selects one mobile robot within a group to establish a communication channel with it. To model the system, the authors used the theory of branching processes (Kendall 1966), which is used in social sciences to model phenomena such as population growth and virus spread.

Finally, Hamann (2012) developed two simple models for swarm robotics systems. In the first, the performance of a generic swarm robotics systems is explained using the interaction between cooperation and interference. In the second, the consensus achievement behavior is studied using a simple probabilistic model based on the urn problem. 


\subsubsection{Real-robot analysis}

The use of real robots (as opposed to simulated robots) to validate a collective behavior is a fundamental tool. In fact, it is practically unfeasible to simulate all the aspects of reality (Frigg and Hartmann 2012; Brooks 1990). Experiments with real robots help to test the robustness of swarm robotics systems that have noisy sensors and actuators. Working with real robots is very important also because it helps discriminating between collective behaviors realizable in practice and those that work only under unrealistic assumptions.

It must be noted that in all real-robot experiments presented in the analyzed literature, the experiments are performed in controlled environments. By controlled environments we mean artificial arenas in which most conditions-e.g., light intensity, radio interference and floor smoothness - can be controlled by the experimenter. This is often very far from the scenarios in which swarm robotics systems are supposed to operate. For this reason, realrobot experiments should not be considered as a way to validate collective behaviors for their use in real-world applications, but rather as a way to test them against realistic noise patterns in sensors and actuators.

In this review we present more than 60 publications dealing with collective behaviors in the swarm robotics field (see Sect. 3). Slightly more than half of these publications presented results obtained only through simulations or models. We believe that the reason behind this choice is that, in general, it is easier, faster, and safer to perform experiments using models or simulations than using robots.

In the papers that included experiments done with real robots, the scope of the use of the robots can be divided in two categories: proof-of-concept experiments and extensive experiments. The first category includes slightly more than half of the analyzed works that involve real-robot experiments. In these works, few runs (typically one) of an experiment with real robots are performed. The aim of real-robot experiments within these works is to show that the proposed collective behavior is realizable. Examples of this kind of experiment can be found in the works by Payton et al. (2001) and Spears et al. (2004). In the other category, instead, several runs are executed and data are gathered to be analyzed for comparison with simulated runs or to show properties of the system. Examples of this kind of experiments can be found in the works by Çelikkanat and Şahin (2010) and O'Grady et al. (2010).

Very often in the swarm robotics literature, the reasons why robots are used are not explicitly stated. In particular, the answer to the question "what does the real-robot study add to the simulation or theoretical study" is almost never explicitly answered, as it should. An effort in this sense would simplify the process of reproducing results as it would help to clarify possible differences between the model and the real-robot system. Moreover, clarifying the role of real robots in experiments can help in porting a similar collective behavior to a different robotic hardware.

\section{Collective behaviors}

In this section, we present a review of the main collective behaviors studied in the literature. These collective behaviors are basic behaviors of a swarm that could be combined to tackle complex real-world applications as, for example, foraging or construction. We classify these collective behaviors into four main categories: spatially organizing behaviors, navigation behaviors, collective decision-making and other collective behaviors.

In the first category, spatially organizing behaviors, we consider behaviors that focus on how to organize and distribute robots and objects in space. In the second category, navigation 
behaviors, we consider behaviors that focus on how to organize and coordinate the movements of a swarm of robots. In the third category, collective decision-making, we consider behaviors that focus on letting a group of robots agree on a common decision or allocate among different parallel tasks. In the last category, other collective behaviors, we consider behaviors that do not fall into any of the categories mentioned above.

For each category, we give a brief description of the collective behavior, its source of inspiration, the most common used approaches and the most significant available results.

\subsection{Spatially-organizing behaviors}

In this section, we describe collective behaviors that focus on how to organize and distribute robots and objects in space. Robots can be organized and distributed in space in several possible ways: aggregates, patterns, chains, and structures of physically connected robots. Moreover, robots can also physically move objects to create clusters and structures.

The simplest spatial organization is the aggregate: a group of robots spatially close to each other. The collective behavior used to obtain an aggregate is called aggregation. Work on aggregation is presented in Sect. 3.1.1.

More complex spatial organizations consist of patterns or chains. Work on pattern and chain formation is presented in Sects. 3.1.2 and 3.1.3.

A different, but related, kind of spatial organization is the one composed by physically connected robots. Collective behaviors used to obtain and manage structures formed by physically connected robots are called self-assembling and morphogenesis behaviors. Work on physically connected robots is presented in Sect. 3.1.4.

Works that explore how robots can collectively organize objects in clusters and assembles are presented in Sect. 3.1.5.

\subsubsection{Aggregation}

Description The goal of aggregation is to group all the robots of a swarm in a region of the environment. Despite being a simple collective behavior, aggregation is a very useful building block, as it allows a swarm of robots to get sufficiently close one another so that they can interact.

Source of inspiration Aggregation is a very common behavior in nature. For example, aggregation can be observed in bacteria, cockroaches, bees, fish and penguins (Camazine et al. 2001). Other examples of natural systems performing aggregation have been described by Grünbaum and Okubo (1994), Breder (1954), Jeanson et al. (2005), and Amé et al. (2006).

Approaches In swarm robotics, aggregation is usually approached in two ways: probabilistic finite state machines (PFSMs) or artificial evolution.

The most common approach is based on PFSMs: the robots explore an environment and, when they find other robots, they decide stochastically whether to join or leave the aggregate. In this approach, a stochastic component is often used in order to ensure that eventually only a single aggregate is formed.

In the artificial evolution approach, the parameters of a neural network are automatically selected in order to obtain an aggregation behavior. 


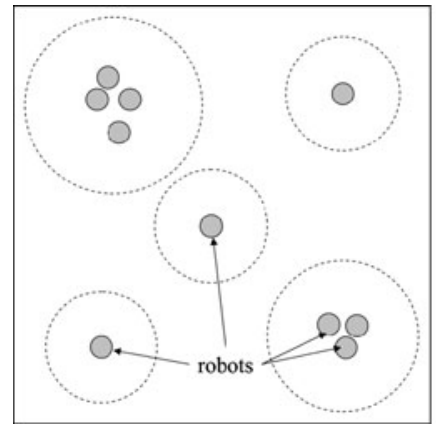

(a) From Soysal and Şahin (2007), reprinted with permission.

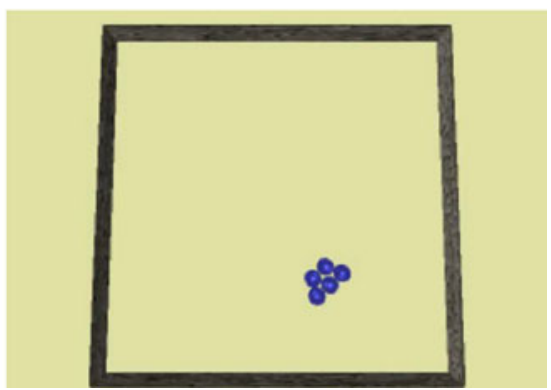

(b) From Trianni et al. (2003), reprinted with permission.

Fig. 4 Examples of the aggregation collective behavior

Results Garnier et al. (2005) developed a system in which robots are used to replicate the behavior observed in cockroaches by Jeanson et al. (2005). The robots are able to collectively aggregate in a circular arena using a PFSM approach.

Another example of an aggregation behavior based on a PFSM was developed by Soysal and Şahin $(2005,2007)$ (see Fig. 4(a)). In their work, a robot can be in one of three states: the repel state, in which the robot tends to get away from other robots; the approach state, in which the robot tends to get closer to other robots; and the wait state, in which the robot stand still. Soysal and Şahin were able to achieve both moving and static aggregation behaviors by changing the parameters of the system.

An example of aggregation obtained with artificial evolution was developed by Trianni et al. (2003) (see Fig. 4(b)). The authors obtained two sets of parameters for a neural network achieving both moving and static aggregates.

Soysal et al. (2007) presented some rules of thumb for obtaining aggregation behaviors through artificial evolution. Moreover, they proposed a comparison between the probabilistic finite state machine approach by Soysal and Şahin (2005) and the artificial evolution approach by Bahçeci and Şahin (2005).

\subsubsection{Pattern formation}

Description Pattern formation aims at deploying robots in a regular and repetitive manner. Robots usually need to keep specific distances between each other in order to create a desired pattern.

Source of inspiration Pattern formation can be found both in biology and in physics. Some biological examples are the spatial disposition of bacterial colonies and the chromatic patterns on some animal's fur (Meinhardt 1982). Some physics examples are molecules distribution and crystal formation (Langer 1980), and Bénard cells (Getling 1998).

Approaches The most common way to develop pattern formation behaviors in robot swarms is to use virtual physics-based design. Virtual physics-based design uses virtual forces to coordinate the movements of robots. 


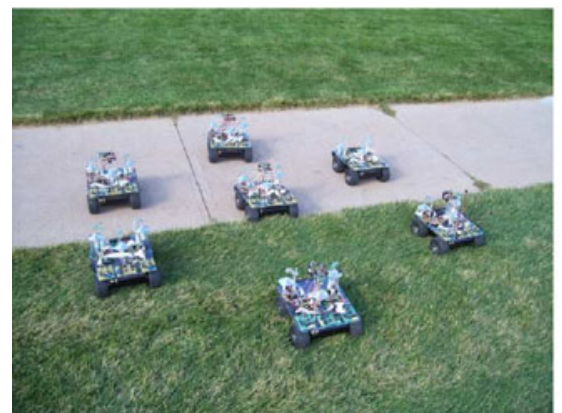

(a) From Spears and Spears (2012) reprinted with permission.

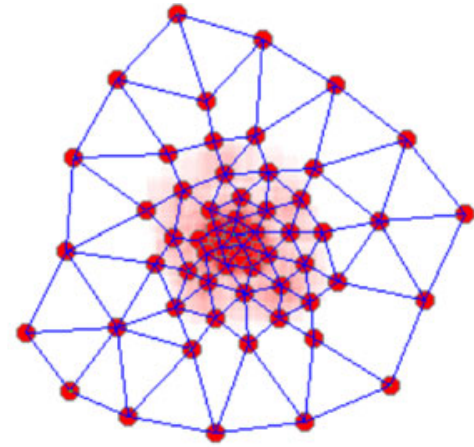

(b) From Shucker and Bennett (2007), reprinted with permission.

Fig. 5 Examples of the pattern formation collective behavior

Results Bahçeci et al. (2003) presented a review of works on pattern formation in which they analyzed centralized and decentralized behaviors. Another review on the topic has been published in 2009 by Varghese and McKee.

Spears et al. (2004) developed a collective behavior for pattern formation that is one of the first applications of virtual physics-based design. In their work, they use the virtual forces to form a hexagonal lattice (see Fig. 5(a)). In the same work, Spears et al. showed that, by creating two groups of robots with different attraction/repulsion thresholds, it is also possible to obtain a square lattice. More details can be found in a subsequent work (Spears and Spears 2012).

Shucker and Bennett (2007) presented a behavior in which robots interact via virtual springs (see Fig. 5(b)). These virtual springs are used by a robot to compute attraction/repulsion virtual forces. Differently from Spears et al.'s work, in this work, the robots can interact in different ways (full connectivity, first neighbors, N-nearest, ....). Each type of interaction has different characteristics and gives rise to slightly different patterns. Additional theoretical work is presented in a subsequent paper (Shucker et al. 2008).

Flocchini et al. (2008) focused on a theoretical analysis of pattern formation. The authors were able to formally prove that with a group of fully asynchronous robots it is possible to obtain only a subset of all possible patterns, whereas other patterns are achievable only with some kind of global knowledge such as a common orientation given by a compass.

\subsubsection{Chain formation}

Description In the chain formation behavior, robots have to position themselves in order to connect two points. The chain that they form can then be used as a guide for navigation or for surveillance.

Source of inspiration The chain formation behavior takes its inspiration from foraging ants. Deneubourg et al. (1990) studied and modeled the behavior of Argentine ants which form chains of individuals connecting their nest with foraging areas.

Approaches Chains of robots can be obtained in multiple ways: the most used design approaches are probabilistic finite state machines (PFSMs), virtual physics-based design and artificial evolution. 


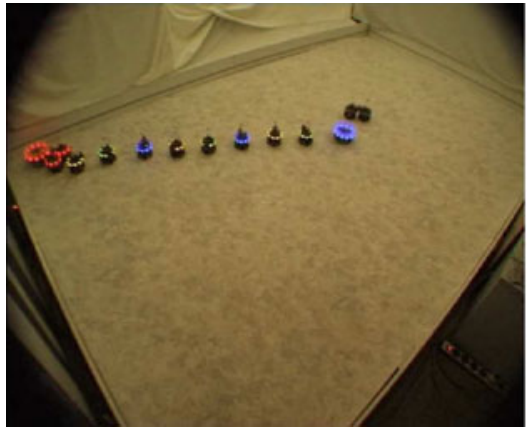

(a) From Nouyan et al. (2009), reprinted with permission.

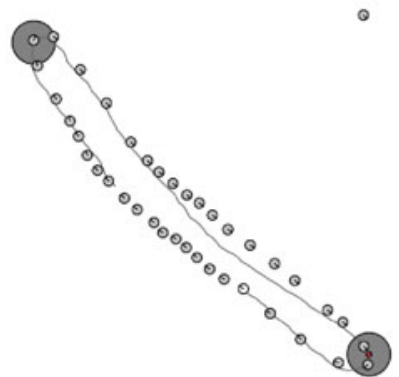

(b) From Sperati et al. (2011), reprinted with permission.

Fig. 6 Examples of the chain formation collective behavior

Results Nouyan et al. (2008, 2009) developed a behavior, based on PFSMs, in which the robots have two different exchangeable roles: explorer and chain member (see Fig. 6(a)). In the explorer role, the robots are searching for chain members or for the goal area. When they find either a chain member or the goal, they switch to the chain member role and stop. Chain members can become explorer again according to a probability that increases over time if no other robots are perceived. Different configurations and approaches are analyzed and presented.

Maxim et al. (2009) used virtual physics-based design to form chains of robots. Virtual forces are used to keep a specific distance between robots and between a robot and the walls of the environment. The developed behavior creates chains that are strongly based on the shape of the environment, which is assumed to be composed of narrow corridors.

Sperati et al. (2011) used artificial evolution to obtain a chain formation behavior (see Fig. 6(b)). In their work, the robots, by using communication through colored LEDs, are able to follow each other forming a double chain between two designated areas. Differently from other chain formation behaviors, in this work the obtained chain is composed of moving robots.

Ducatelle et al. (2011a) proposed a collective behavior based on PFSMs and network routing which was able to obtain a chain composed of moving robots. The details of this work are presented in Sect. 3.2.1.

\subsubsection{Self-assembly and morphogenesis}

Description In robotics, self-assembly is the process by which robots physically connect to each other. Self-assembly can be used for different purposes. For example, to increase stability when navigating on rough terrains or to increase the pulling power of the robots. Morphogenesis is the process that leads a swarm of robots to self-assemble following a particular pattern, and can be used by the swarm to self-assemble into a structure that is particularly appropriate for a given task. For example, self-assembling into a line can allow to pass on a narrow bridge, while a blob-like shape will make moving on rough terrain more stable.

Source of inspiration Self-assembly can be observed in several species of ants. Ants are able to physically connect in order to perform different tasks. Some examples of structures 


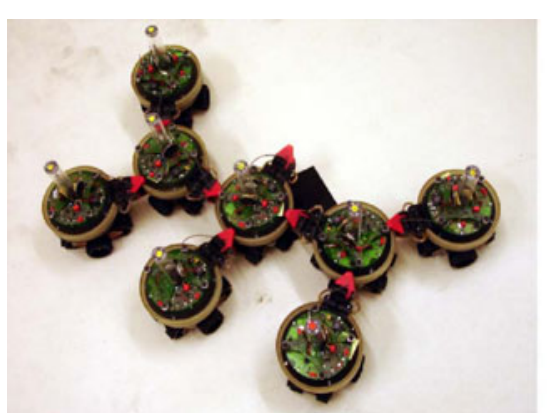

(a) From Christensen et al. (2008), reprinted with permission.

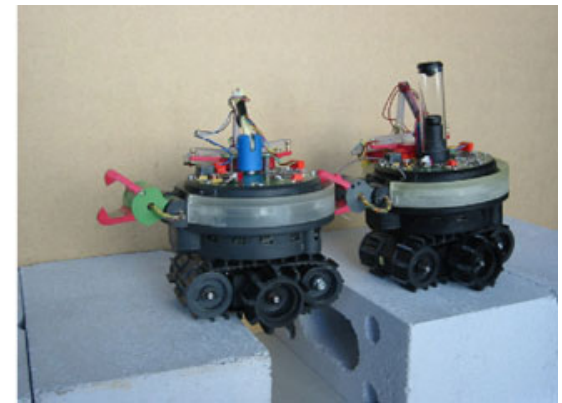

(b) From Mondada (2005), reprinted with permission.

Fig. 7 Examples of the self-assembly collective behavior

created by ants are bridges, rafts, walls and bivouacs (Anderson et al. 2002). Self-assembly and morphogenesis are studied also by developmental biology: scientists study how cells develop and self-organize to form tissues and organs (Turing 1953).

Approaches From the swarm robotics perspective, there are two main challenges: how to self-assemble into a desired target structure (i.e., morphogenesis), and how to control the obtained structure to tackle specific tasks. Works focusing on the first issue are usually based on probabilistic finite state machines and rely on communication for coordination. Works focusing on the second issue, make use either of artificial evolution or of probabilistic finite state machines.

Results A review of the literature on self-assembly and morphogenesis has already been presented by Groß and Dorigo (2008b). For this reason, in this paper, we discuss only some examples of recent works.

O'Grady et al. (2009a) presented a morphogenesis behavior for self-assembling robots that are able to signal docking points on their body to other robots using LEDs. Different structures, such as lines, stars and circles, can be obtained by having the robots signal docking points in different positions (see Fig. 7(a)). A related scripting language for the morphogenesis process has been presented by Christensen et al. (2008). Both works were realized in the context of the Swarm-Bots project (Dorigo et al. 2006).

Results on the control aspect of self-assembly depend strongly on the goal of the system considered. O'Grady et al. (2010) demonstrated that physically connected robots can navigate through difficult terrains better than robots that are not connected. In O'Grady et al.'s work, robots randomly explore an environment with slopes. Each robot is able to measure the steepness of these slopes and when a slope is steeper than a certain threshold, it can initiate a self-assembling procedure. Once connected into a structure, the robots can navigate in hazardous terrains thanks to the high mechanical stability given by the new morphology. Mondada et al. (2005) showed that physically connected robots are able to cross a ditch that is too large for a single robot to overcome (see Fig. 7(b)). Finally Groß and Dorigo (2009) showed that physically connected robots are able to obtain better results, in terms of speed and distance, in the transportation of heavy objects compared to non-connected robots.

The Symbrion and Replicator projects tackled both the morphogenesis and the control aspects of self-assembly (Levi and Kernbach 2010). In these projects, swarms of selfassembling robots capable of creating 3D structures are studied. Such robots are able, when connected, to share energy and computational resources with their neighbors. 
Another aspect of self-assembly is how to make the swarm decide who should assemble with whom. In the work of Ampatzis et al. (2009), a duo of robots has to assemble to each other without prior knowledge of who will grip and who will be gripped. The authors proposed a solution based on artificial evolution and recurrent neural networks which can make time-dependent decisions. Mathews et al. (2012) used a heterogeneous approach: a flying robot is used to recognize the task to tackle and guide ground-based robots. The flying robot communicates to the ground-based robots which robots should self-assemble and what kind of structure to create to tackle the task.

\subsubsection{Object clustering and assembling}

Description In this section we present works in which robots move objects spread in an environment. The robots can follow two kinds of behavior: clustering and assembling. The goal of object clustering and assembling is to group objects close one to the other. The difference between clusters and assembles is that clusters are composed of non-connected objects, whereas assembles are composed of physically linked objects. The object clustering and assembling behaviors are fundamental components of any construction process.

Source of inspiration The object clustering and assembling behaviors are displayed by many social insects. For example, ants exhibit brood clustering (Franks and Sendova-Franks 1992) and termites are able to deposit mud to build complex nests (Grassé 1959). Insects usually exploit natural occurring gradients, such as temperature gradients, or pheromones gradients to tackle this tasks.

Approaches In swarm robotics, object clustering and assembling are usually approached using probabilistic finite state machines. The robots explore the environment at random and react in different ways to the discovery of available objects or of part of the cluster/assemble to create.

In almost all analyzed works, robots group objects sequentially or quasi sequentially. In fact, parallelism could potentially create collisions and interference and thus is avoided. To avoid such problems, a robot usually prevents other robots from depositing objects at the same time, either by using communication or by physically blocking access to the cluster site.

Object aggregates are usually obtained using blocks with some kind of self-alignment mechanism based, for instance, on magnets.

Results One of the pioneering work in object clustering is the one by Beckers et al. (1994). In this work, the robots follow a very simple behavior: they explore the environment at random and, when they find an object, they pick it up. A robot with an object moves at random in the environment and deposits the object with a probability proportional to the number of other objects observed. Following these simple rules, the robots are able to create clusters of objects.

Melhuish et al. (1999b) presented a work in which robots create clusters of object roughly in the shape of a wall (see Fig. 8(a)). In this work, disks are scattered around the environment and a specific area is marked for disk clustering. Such area is located half way between a light and a line on the ground. The robots measure the distance between the line on the ground and the light to recognize the clustering area. The robots follow simple reactive rules to cluster the objects roughly in the shape of a wall. In a following work, Stewart and Russell (2006) developed a similar mechanism in which the position of the cluster is marked by a moving robot instead of a fixed light. 


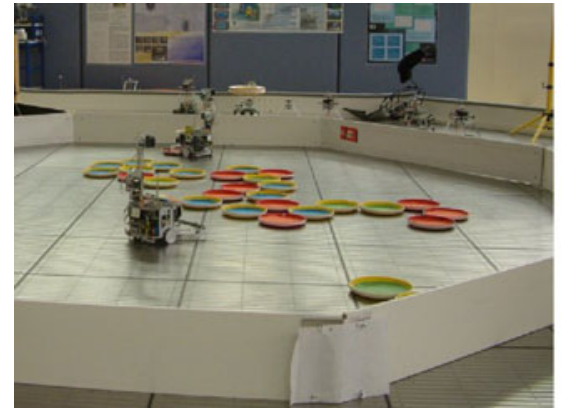

(a) From Melhuish (1999), reprinted with permission.

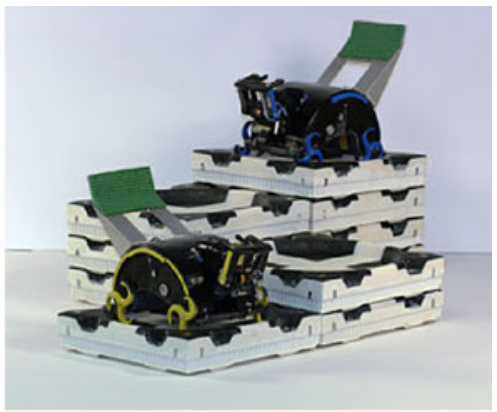

(b) From Werfel (2011), reprinted with permission.

Fig. 8 Examples of the object clustering and assembling collective behavior

Wawerla et al. (2002) developed a behavior to create simple 2D walls made of blocks of alternating color. A robot performs random walk in search for a block. After collecting a block, the robot searches either for the seed block or for the partially constructed wall. When it finds the wall or the seed block, the robot checks if no other robot is already placing a block by using local communication, and then places the blocks.

Werfel (2006) developed a method for creating arbitrary 2D structures with blocks placed over a virtual grid. In this work, all robots have knowledge of a matrix which encodes the final structure to create. Such plan is used by the robots also as a frame of reference. The idea is the following: when a robot finds part of the structure, it follows it counting the placed blocks. This block-counting behavior allows the robot to locate itself in the frame of reference of the structure to create. Once the robot knows its position with respect to the placed blocks, it can decide where to place the next block using its knowledge of the final structure. This approach was also extended to create 3D structures (Werfel and Nagpal 2008) and tested using real robots (Werfel et al. 2011) (see Fig. 8(b)).

\subsection{Navigation behaviors}

In this section, we describe collective behaviors that cope with the problem of coordinating the movements of a swarm of robots. In Sect. 3.2.1, we review works on collective exploration. Collective exploration is a collective behavior in which robots cooperate to explore an environment and perform navigation. In Sect. 3.2.2, we review works on coordinated motion. The coordinated motion behavior is used to make robots move together like a flock of birds or a school of fish. In Sect. 3.2.3, we review works on collective transport. In the collective transport behavior, robots cooperate to transport an object that is too heavy to be moved by a single robot.

\subsubsection{Collective exploration}

Description In this section, we analyze two collective behaviors that, together, can be used to achieve collective exploration of an environment: area coverage and swarm-guided navigation. The goal of area coverage is to deploy robots in an environment in order to create a regular or irregular grid of communicating robots. The obtained grid can be employed, for example, to monitor the environment for hazardous leaks or to guide other robots. We call the behavior necessary to guide the navigation of other robots swarm-guided navigation. Since the two behaviors are strongly linked, many works focus on both at the same time. 


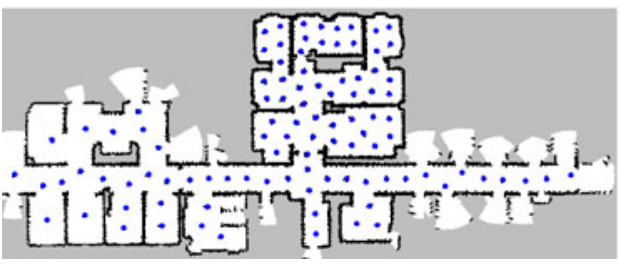

(a) From Howard et al. (2002), reprinted with permission.

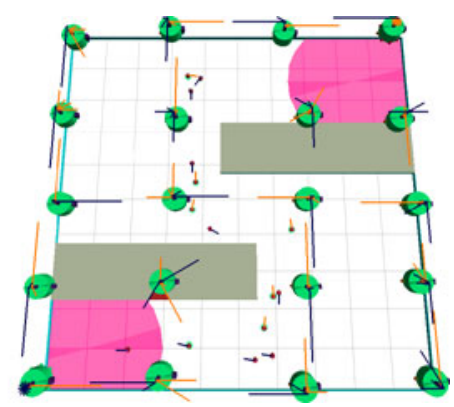

(b) From Ducatelle et al. (2011b), reprinted with permission.

Fig. 9 Examples of the collective exploration behavior

Source of inspiration Area coverage and navigation are common behaviors of social animals. For example, ants use pheromone trails to find the shortest route between two points and bees directly communicate destinations in the environment by means of dances (Camazine et al. 2001). Area coverage has been intensively studied also by the wireless sensor networks (WSN) community. A survey of area coverage behaviors in WSN was conducted by Wang et al. (2009).

Approaches In swarm robotics, the most common way to tackle area coverage is to use virtual physics-based design to obtain a grid covering the environment. Works on swarmguided navigation instead focus on communication, thus usually employ probabilistic finite state machines and take inspiration either from network routing protocols or natural systems.

Results Payton et al. (2001) used robots as "virtual pheromones". Some robots, which are already deployed, are able to create a gradient between the source and the target by exchanging messages. This gradient can then be exploited for navigation by other robots or by a human.

Howard et al. (2002) developed a behavior using virtual physics-based design. Each robot is repelled by other robots and by obstacles. This approach allows the robots to maximize the area covered and form a connected communication network (see Fig. 9(a)).

O'Hara and Balch (2007) presented a behavior that exploits pre-deployed sensors to perform foraging in an environment that can change over time. Through a distributed BellmanFord algorithm, a navigation route towards a specific goal is found and then followed by the mobile robots.

Nouyan et al. (2009) used chain formation (see Sect. 3.1.3) to link an object in the environment with the robot nest. The robots in the chain display a pattern of repeating colors to indicate in which direction is the nest and in which direction is the object. This information is used by the other robots to navigate the environment.

Di Caro et al. (2009) presented a work in which robots are able to navigate from a source to a target location. The proposed behavior is based on communication with other passive robots already available in the environment. These passive robots are assumed busy with other collective behaviors but are able to guide the navigating robots.

Stirling and Floreano (2010) used a swarm of flying robots to achieve area coverage. In their work, the robots are deployed sequentially and each robot determines its position according to the position of the previously deployed robots. Only one or few robots, called 
explorers, are flying at the same time, whereas the great majority is attached to the ceiling and act as communication relay. One particular aspect of Stirling and Floreano's approach is the ability to explore an environment with a limited number of robots, as the robots can leave an area once it has been visited. This work was developed for the Swarmanoid project (Dorigo et al. 2012).

Ducatelle et al. (2011a) proposed a collective behavior based on network routing, capable of guiding a robot from a source area to a target. Similarly to what happens in packet routing, the robots keep a table of the distance of other robots with respect to the target. A robot can then use the entries in the table and reach the target. Ducatelle et al. studied two different experimental setups. In the first, only a single robot is moving while the others act as beacons to guide it. In the second, all the robots are moving between two points, creating a dynamic chain.

Ducatelle et al. (2011b) studied collective exploration using a heterogeneous robotic swarm (see Fig. 9(b)). They tackled an indoor navigation task, in which a swarm of wheeled robots move back and forth between a source and a target location. The path of the wheeled robots are guided by a swarm of flying robots that can attach to the ceiling and overview the progress of the wheeled robots. Their solution is based on mutual adaptation: wheeled robots execute instructions given by flying robots, and flying robots observe the behavior of wheeled robots to adapt their position and the instructions they give. Ducatelle et al. developed the system using a probabilistic finite state machine together with techniques from network routing protocols.

\subsubsection{Coordinated motion}

Description In coordinated motion, also known as flocking, robots move in formation similarly to schools of fish or flocks of birds. For a group of autonomous robots, coordinated motion can be very useful as a way to navigate in an environment with limited or no collisions between robots and as a way to improve the sensing abilities of the swarm (Kaminka et al. 2008).

Source of inspiration Coordinated motion behaviors are frequent in almost all social animals. In particular, flocking in group of birds or schooling in group of fish are impressive examples of self-organized coordinated motion (Okubo 1986). Through coordinated motion, animals gain several advantages, such as a higher survival rate, more precise navigation and reduced energy consumption (Parrish et al. 2002).

Approaches In swarm robotics, coordinated motion behaviors are usually based on virtual physics-based design. Robots are supposed to keep a constant distance from one another and an uniform alignment while moving (Reynolds 1987b). Coordinated motion behaviors have also been obtained via artificial evolution.

Results The first work on coordinated motion was published by Reynolds (1987b) in the domain of computer graphics. Reynolds developed a flock of virtual birds in which the individuals are able to sense the velocity and the range of the neighbors. The individuals follow three simple rules: collision avoidance, velocity matching and flock centering (see Fig. 10(a)). Collision avoidance keeps the individuals from colliding one with the other. Velocity matching ensures that each individual matches the speed of its neighbors and flocking centering forces each individual to stay close to its neighbors.

Balch and Hybinette (2000) proposed a coordinated motion behavior based on social potentials. Each robot knows the position and orientation of the robots in its sensing range 


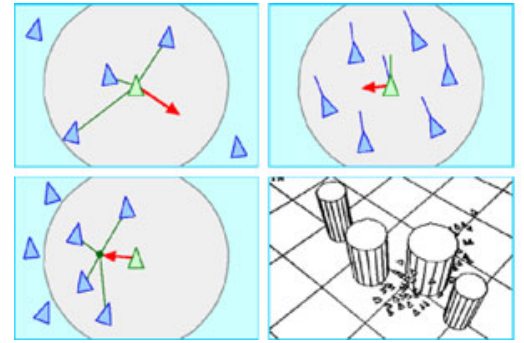

(a) From Reynolds (1987a), reprinted with permission.

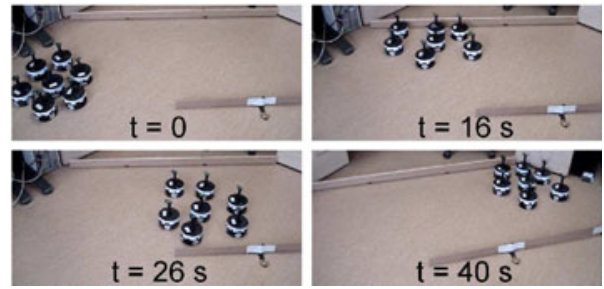

(b) From Turgut et al. (2008a), reprinted with permission.

Fig. 10 Examples of the coordinated motion behavior

and thus it is able to compute the target position to reach. The authors created a coordinated motion behavior that is able to avoid obstacles and form different patterns, such as lines, diamonds and squares.

Baldassarre et al. (2003) used artificial evolution to tune the parameters of a neural network in order to perform coordinated motion. The authors were able to obtain three coordinated motion behaviors. These behaviors differ by how each robot moves with respect to the others. In the first behavior, the robots keep a constant speed. In the second one, only one robot moves, while the rest of the swarm tries to remain close to it. In the last behavior, the robots rotate around the center of the swarm.

Turgut et al. (2008a) developed a virtual heading sensor which allows each robot to sense the heading direction of the other robots. With this information and knowing the distance of the neighbors (by means of an infrared sensor), the swarm was able to obtain coherent coordinated motion and obstacle avoidance in absence of a common goal direction (see Fig. 10(b)). The developed behavior is one of the first true implementation of Reynolds' (1987b) flocking behavior with real robots. The authors evaluated the performance of their behavior by using different metrics and validated it with the use of several robots.

Çelikkanat and Şahin (2010), extending the work of Turgut et al. (2008a), showed that it is possible to insert some "informed" robots in the swarm in order to direct the movement of other "non-informed" robots. The informed robots are the only ones in the group with knowledge of the goal direction. Increasing the number of informed robots or decreasing the individual tendency to follow other robots increase the accuracy of motion of the group with respect to the desired goal direction. These works have been extended by Ferrante et al. (2010) who developed alternative communication strategies in which some robots explicitly communicate their headings.

Stranieri et al. (2011) first introduced the idea of coordinated motion without the need for all robots to perceive the orientation of their neighbors. Their work has been extended by Ferrante et al. (2012), thus we present in detail only this most recent work.

Ferrante et al. (2012) proposed a coordinated motion behavior that, differently from other works, does not require an explicit alignment rule: the robots in the swarm use only attraction and repulsion rules. The key difference is in the novel way in which the robots translate the vector computed using the attraction and repulsion rules into wheel actuation. In fact, in all previous works, the robots changed their angular speed according to the direction of this vector while keeping their forward velocity fixed. In this work instead, the robots change also their forward speed, according to the magnitude of the computed vector. Additionally, the authors showed that the swarm is able to navigate both with and without the presence of informed robots, that is, robots that know the desired direction to follow. 


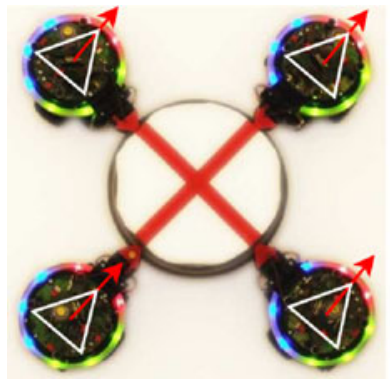

(a) From Campo et al. (2006), reprinted with permission.

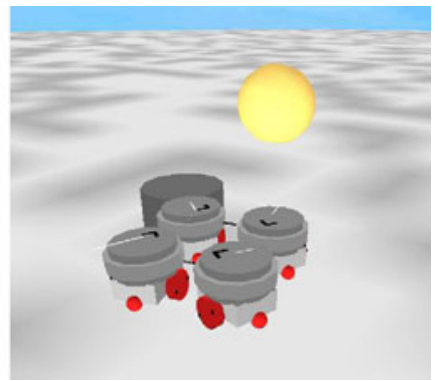

(b) From Baldassarre (2006), reprinted with permission.

Fig. 11 Examples of the collective transport behavior

\subsubsection{Collective transport}

Description Collective transport, also known as group prey retrieval, is a collective behavior in which a group of robots has to cooperate in order to transport an object. In general, the object is heavy and cannot be moved by a single robot, making cooperation necessary. The robots need to agree on a common direction in order to effectively move the object towards a target.

Source of inspiration Ants often carry prey cooperatively. Kube and Bonabeau (2000) analyzed how cooperative transport is achieved in ant colonies. When ants find their target, they physically attach to it and then start to pull and push. If they do not perceive any movement after a while, they change the orientation of their body and try again. If even this does not work, they detach, re-attach at a different point and try again.

Berman et al. (2011a) studied the same behavior observing how ants interact with fabricated elastic structures. Using the data retrieved from these observations, the authors developed a mathematical model of how collective transport is performed by ants.

Approaches In swarm robotics, collective transport behaviors are obtained by using probabilistic finite state machines or artificial evolution. Cooperation is obtained either through explicit communication of the desired motion direction, or through indirect communication, that is, by measuring the force applied to the carried object by the other robots.

Results Donald et al. (1997) proposed three behaviors based respectively on: force sensing, position sensing and orientation sensing. This work was one of the first works aimed at studying collective transport without a centralized controller and with limited communication.

Campo et al. (2006) proposed a collective behavior in which the robots end up moving in a common direction at the beginning of the experiment by communicating their individual direction (see Fig. 11(a)). In this way the robots are able to drag an object towards a goal area, even if this area is not perceived by all robots.

Groß and Dorigo (2009) used artificial evolution to tune the parameters of a neural network to achieve collective transport. The obtained behavior was able to cope with different prey sizes and prey weights as well as with different numbers of robots (from 4 to 16). Various metrics and extensive simulations were used to validate the results. In their work, Groß 
and Dorigo were able to obtain three different transport strategies. In the first, the robot directly connects to the prey and move it. In the second, the robots connect to each other and then to the prey to move it. In the third and last strategy, the robots are not directly connected to the prey but form a circle around the prey and push it.

Baldassarre et al. (2006) used artificial evolution and neural networks to perform collective transport (see Fig. 11(b)). The obtained behavior exploits a sensor able to perceive the force applied by other robots on the chassis. With this sensor, the robots are able to perform collective obstacle avoidance while going towards a target area.

Ferrante et al. (2013) developed a collective transport behavior in which, through communication, a group of robots can agree on a common moving direction towards a goal by averaging the individual desired direction. The proposed solution is able to make robots move towards a common goal while avoiding obstacles. This work was developed for the Swarmanoid project (Dorigo et al. 2012).

\subsection{Collective decision-making}

Collective decision-making deals with how robots influence each other when making choices. It can be used to answer two opposite needs: agreement and specialization. A typical example of agreement in swarm robotics systems is consensus achievement. The desired outcome of consensus achievement is that all the robots of the swarm eventually converge towards a single decision among the possible alternatives. A typical example of specialization, instead, is task allocation. The desired outcome of task allocation is that the robots of the swarm distribute themselves over the different possible tasks in order to maximize the performance of a system.

\subsubsection{Consensus achievement}

Description Consensus achievement is a collective behavior used to allow a swarm of robots to reach consensus on one choice among different alternatives. The choice is usually the one that maximize the performance of the system. Consensus is generally difficult to achieve in swarm of robots due to the fact that very often the best choice may change over time or may not be evident to the robots due to their limited sensing capabilities.

Source of inspiration Consensus achievement is displayed in many insect species. For example, ants are able to decide between the shortest of two paths using pheromones (Camazine et al. 2001). Bees have mechanisms to collectively decide which is the best foraging area or which is the best nest location among several possibilities (Couzin et al. 2005). These mechanisms work even if not all the individuals in the swarm have an opinion on the best choice. Cockroaches also display consensus achievement behaviors when performing aggregation (Amé et al. 2006).

Approaches In swarm robotics, the approaches used for consensus achievement can be divided into two categories according to how communication is used. In the first category, direct communication is used: each robot is able to communicate its preferred choice or some related information. In the second category, instead, indirect communication is used: the decision is performed through some indirect clues, such as the density of the robot population. 


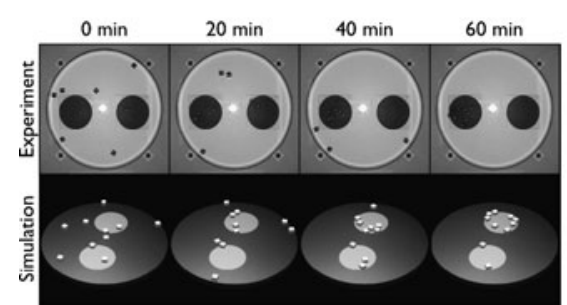

(a) From Garnier et al. (2005), reprinted with permission.

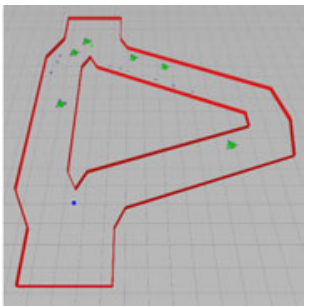

(b) From Montes de Oca et al. (2011), reprinted with permission.

Fig. 12 Examples of the consensus achievement collective behavior

Results Wessnitzer and Melhuish (2003) proposed a collective behavior in which robots "hunt" two moving targets. The robots decide which target to follow first, follow it and block it. They then do the same for the second target. Two consensus achievement behaviors are proposed: in the first, the robots simply follow the robot closest to a target, resulting in a decision based on the spatial distribution of the swarm; in the second, the robots vote, using a majority rule, to decide which target to follow.

Garnier et al. $(2005,2009)$ studied consensus achievement in cockroaches by using a swarm of robots to replicate the experiment by Amé et al. (2006) (see Fig. 12(a)). In their system, consensus achievement is obtained through indirect communication. The focus of this work is both on consensus achievement and aggregation. For this reason this work was presented also in Sect. 3.1.1. A mathematical model of the same behavior was developed by Correll and Martinoli (2007). In a similar work, Campo et al. (2011) presented a collective behavior in which the swarm aggregates on the smallest resource that can host the whole group. A further extension was proposed by Francesca et al. (2012). The authors used evolutionary robotics to replicate the results obtained by Amé et al. (2006) comparing the two works also using a macroscopic model.

Gutiérrez et al. (2010) developed a strategy for consensus achievement through direct communication in a swarm of robots performing foraging. The robots are able to decide between two foraging areas. When two robots get close, they exchange their measured distances between the nest and the latest visited goal. Each robot performs an average of its measured distance with the one received from the other robots. In this way, the robots are able to agree on which area is the closest to the nest and discard the other one even when the measured distances are noisy.

Parker and Zhang (2011) proposed a consensus achievement behavior based on quorum sensing. The algorithm is inspired by how ants and bees choose the best nest over $\mathrm{N}$ alternatives (Couzin et al. 2005). When a robot finds a new alternative, it evaluates its quality and sends recruiting messages to other robots to advertise it. The frequency of these messages is proportional to the perceived quality of the alternative. Thanks to the different message frequencies associated with the different alternatives, over time all robots converge on the best alternative. The behavior is implemented as a probabilistic finite state machine.

Montes de Oca et al. (2011) focused on consensus achievement in a system where robots are performing multiple parallel executions of collective transport in groups of three from a nest area to a goal area. The robots need to reach consensus between two possible paths, one longer than the other (see Fig. 12(b)). Each individual robot has a preferred path. When a group of three robots is formed in the nest, the robots choose the path that is preferred by the majority of them. The chosen path becomes the preferred one for all the robots in the group. 
Since the robots choosing the short path take less time to complete the execution, they are more often in the nest. This results in more groups formed by robots preferring the short path than those preferring the long path. This asymmetry eventually makes the robots use the shortest path. A mathematical model of this system was presented by Scheidler (2011). In this work, consensus is achieved both through direct and indirect communication, as the robots use direct communication at group level, and indirect communication at the swarm level.

\subsubsection{Task allocation}

Description Task allocation is a collective behavior in which robots distribute themselves over different tasks. The goal is to maximize the performance of the system by letting the robots dynamically choose which task to perform.

Source of inspiration Task allocation can be observed in natural systems such as ant and bee colonies-e.g., Theraulaz et al. (1998). For example, in ant or bee colonies, part of the swarm can perform foraging while another part looks after the larvae. Task allocation is not fixed but can change over time.

Approaches In swarm robotics, task allocation is mainly obtained through the use of probabilistic finite state machines. To promote specialization, the probabilities of selecting one of the available tasks are either different among the robots or they can change in response to task execution or messages from other robots. In swarm robotics, task allocation has been studied mainly on robots performing foraging.

Results In one of the first works on task allocation, Krieger and Billeter (2000) developed a very simple, threshold based mechanism. Robots have to collect prey that are then converted into energy in the nest. While foraging, the robots consume energy. To replenish this energy, the robots can draw it from a common reservoir. Each robot decides to leave and collect prey or to stay in the nest according to a probability. This probability depends on whether the nest energy is above or below a given threshold. Since this threshold is not homogeneous in the swarm, the number of robots allocated to foraging or to resting is a function of the energy level of the nest.

Agassounon and Martinoli (2002) studied task allocation in a foraging task similar to the one studied by Krieger and Billeter (2000). However, in this case the probability to select the foraging task or the resting task depends on individual observations of the environment and of other robots. Thus, the probability is a function of the success or failure of the last foraging trial, of the frequency with which other robots are encountered when foraging or of the perceived density of prey. A mathematical model of a similar task allocation behavior has been developed by Liu et al. (2007) (see Fig. 13(a)).

Yun et al. (2009) studied the problem of how to allocate robots on a construction site so that the number of assembling operations to do is shared equally. Each robot computes optimal equal-mass partitions, that is, partitions with the same number of operations, by sharing information with its neighbors. The developed behavior is robust to changes in the environment and scalable with the number of robots.

Pini et al. (2009) developed a task allocation behavior in a swarm of robots performing foraging using a bucket-brigade approach. In this work, the experiment arena is divided in three areas, the first one is the nest, the second one is an exchange area and the third one is where the prey are. In the exchange area, the robots have the possibility to wait for 


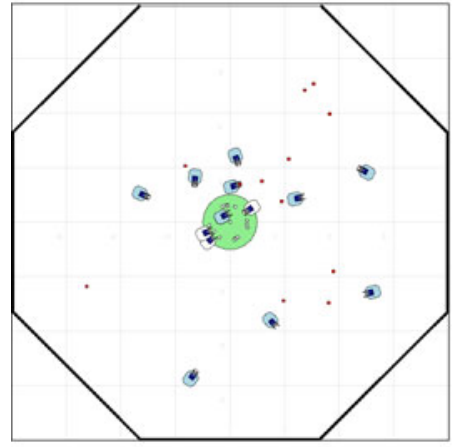

(a) From Liu (2007), reprinted with permission.

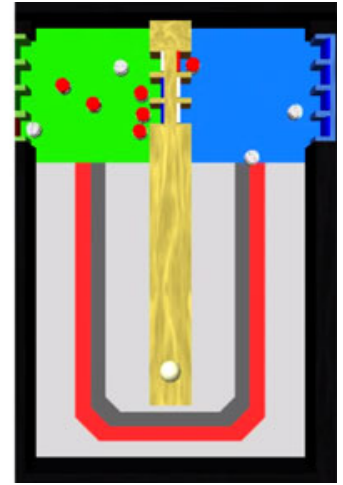

(b) From Pini (2011), reprinted with permission.

Fig. 13 Examples of the task-allocation collective behavior

other robots in order to exchange the prey in a bucket-brigade fashion. Through different thresholds on the waiting time, the robots autonomously change their role between those who bring the prey from the source to the exchange area and those who bring the prey from the exchange area to the nest.

Halász et al. (2012) studied task allocation using robots performing stick pulling. Robots must remove sticks scattered in the environment. To remove a stick, two robots must cooperate performing two parts of the task: one holds a stick from the top and the other from the bottom. Once a robot finds a stick, it holds its top and waits for another robot to complete the second part of the task. If after a certain waiting time no one helped, the robot leaves the stick and searches for another one. This waiting time is changed dynamically according to how well a robot performed in the past. Although the results were not conclusive, the authors observed that, over time, the robots develop a preference for one of the two parts of the tasks, specializing in robots holding the top part of a stick and robots holding the bottom part.

Pini et al. (2011) considered a situation in which robots can choose between carrying a prey directly from the source to the nest and storing it in a dedicated two-sided structure called TAM (Brutschy et al. 2012) (see Fig. 13(b)). Stored prey can be collected by robots waiting on the other side of the structure and carried to the nest. The authors develop a mechanism that allows the robots to choose whether to use the structure on the basis of the cost involved.

\subsection{Other collective behaviors}

In this section, we present some works in swarm robotics that we consider significant but that do not fall in any of the categories presented above.

\subsubsection{Collective fault detection}

Autonomous robots have still a limited reliability. Even though the quality and robustness of the hardware is increasing, hardware failures are still quite common. Techniques to allow robots to autonomously detect failures and faulty behaviors have been developed by exploiting the natural redundancy of swarm robotic systems. 
Christensen et al. (2009) developed a swarm level fault detection behavior based on fireflies synchronization. All the robots in the swarm are emitting a signal in a synchronous way. The robots are able to perceive if another robot is in a faulty state by observing if it is synchronized with them. If a robot is not synchronized, it is assumed to be faulty and a response is initiated.

\subsubsection{Group size regulation}

Group size regulation is the collective capability of creating or selecting a group of a desired size. This can be useful for many reasons. For example, Lerman and Galstyan (2002) showed how an excessive number of robots can reduce the performance of a system, and demonstrated for different behaviors that it is possible to identify a group size that maximizes the performance of the swarm.

Melhuish et al. (1999a) used behavior inspired by fireflies to achieve the formation of groups of the desired size. Each robot can emit, at a random time, a signal. The robots then count the number of signals received over a period. The obtained number can be used by the robots to estimate the size of the group and thus to create groups of the desired size. In a related work, Brambilla et al. (2009) improved the original behavior by introducing a more strict signaling order. With this improvement the authors were able to obtain a more robust and reliable estimate of the size of the group.

Pinciroli et al. $(2009,2010)$ studied a collective behavior able to form groups of robots of the desired size. The swarm is composed of flying robots and terrestrial moving robots. The ground robots perform aggregation under the flying robots. The probabilities used by them to join or leave a group are communicated by the flying robots according to the size of the group itself. With this simple mechanism the robots are able to form groups of various sizes.

\subsubsection{Human-swarm interaction}

Swarm robotics systems are conceived to be autonomous and to make decisions in a distributed way. While these are in general considered to be positive features, they also limit the degree of control of a human operator over the system. In fact, since there is neither a leader nor centralized control, the operator does not have a simple way to control the behavior of the system. Human-swarm interaction studies how a human operator can control a swarm and receive feedback information from it.

McLurkin et al. (2006) developed a simple mechanism in which robots are able to provide information to human operators using LEDs and sound.

Naghsh et al. (2008) proposed an analysis of different possible approaches to humanswarm interaction, classifying them in direct human-swarm interaction, direct swarmhuman interaction, and remote interaction via base station.

Podevijn et al. (2012) used a Microsoft Kinect system to give commands through gestures to a swarm of robots. The human operator is able to command the swarm to select, split, merge, and rotate.

Giusti et al. (2012) used a similar approach based on gestures. The robots observe the gestures of a human operator. Each robot is able to guess the performed gesture, but due to its limited vision capabilities, different robots could make a different guess. To reach consensus, the robots vote using multi-hop communication and finally execute the order associated with the performed gesture.

Kolling et al. (2012) presented two approaches to control a swarm. The first approach is based on global communication: a human operator uses a central computer to select and 
control a subgroup of robots. The second approach is based on local interactions: the human operator places pre-programmed beacons in the environment. Such beacons are used to communicate a new behavior to the robots which are in their communication range.

\section{Conclusions: limits and future directions}

Swarm robotics is an approach to collective robotics that has received a great deal of attention in recent years. Swarm robotics aims at developing systems that are robust, scalable and flexible. In this paper, we analyzed the literature from the swarm engineering perspective. We proposed two taxonomies to analyze the swarm robotics literature: the methods taxonomy, in which we discussed the main design and analysis methods, and the collective behaviors taxonomy, in which we categorized the main collective behaviors according to their goal.

Swarm robotics has several possible applications, including: exploration, surveillance, search and rescue, humanitarian demining, intrusion tracking, cleaning, inspection and transportation of large objects. Despite their potential to be robust, scalable and flexible, up to now, swarm robotics systems have never been used to tackle a real-world application and are still confined to the world of academic research. At the current state of development of the swarm robotics field, the focus is mostly on obtaining desired collective behaviors and understanding their properties. In order to avoid the problems that arise in real-world applications, researchers usually tackle a simplified testbed application.

Foraging is the most used testbed application for swarm robotics systems. Robots have to retrieve "prey" objects from an environment and bring them back to a "nest". Foraging, while simple, can be considered as an abstraction with many points in common with more complex applications, such as demining and search and rescue. Foraging is also used to investigate the effect of interference in swarm robotics systems (Lerman and Galstyan 2002). In particular, foraging is commonly used as a testbed for collective exploration (see Sect. 3.2.1), collective transport (see Sect. 3.2.3) and collective decision-making (see Sect. 3.3).

Another testbed application that has attracted a lot of interest recently is construction. Swarms of robots could be used to build complex structures in those cases in which humans would be unable to, such as underwater and in space. Moreover, construction could greatly benefit from the parallelism and flexibility of swarm robotics systems. Construction is a complex task that requires the combination of several collective behaviors, such as object clustering and assembling (see Sect. 3.1.5) to assemble material, collective transport (see Sect. 3.2.3) to carry material, and collective decision-making (see Sect. 3.3) to allocate the robots to the different sub-tasks of the construction process. A recent example of construction performed by flying robots can be found in the work by Lindsey et al. (2012). Even though this construction system cannot be considered a swarm robotics system, as the robots exploit a centralized system for localization and action planning, the presented problem can be considered as an interesting testbed for swarm robotics systems.

There are many possible reasons for the absence of robot swarms in the real world, such as, for instance, the hardware limitations of the available robots. We foresee that, in the near future, swarm robotics will be used more and more frequently to tackle real-world applications. With an increasing use of swarm robotics systems, we envision an increasing need for a swarm engineering, that is, a need for methods for: (1) requirement modeling and specification, (2) design and realization, (3) verification and validation, and (4) operation and maintenance. In the following, we analyze how these aspects of swarm engineering have been tackled and we discuss some open problems. 
Requirement modeling and specification With the application of swarm robotics to realworld scenarios, we foresee an increasing need for well-defined processes to help in requirement gathering and for formal languages to help in requirement specification. Up to now, none of these processes have been studied directly in swarm robotics. This is probably due to the lack of real-world applications. Effort will therefore be necessary to understand whether existing requirement gathering processes and existing requirement specification languages from other fields can be re-used or adapted for swarm robotics or whether new ones need to be developed.

Design and realization The design aspect of swarm engineering was discussed thoroughly in Sect. 2.1. One main issue remains open: the lack of methods for the top-down design of collective behaviors. Automatic design methods can be considered as top-down methods because, in theory, the process is driven by the global goal desired from the system (see Sect. 2.1.2). However, even if these methods are improving, a lot of domain knowledge is still required to tackle medium to complex applications. In the last years, some top-down methods have been proposed (see Sect. 2.1.1). These methods have shown limited applicability at the current stage, since they have been used only for very specific problems. Moreover, it is not clear how well they can be extended to different scenarios or robots.

Verification and validation Verification and validation exploit analysis methods, which have been discussed in detail in Sect. 2.2. Despite the great number of analysis methods, performing verification and validation of a swarm robotics system and comparing one system with another are still very difficult tasks. The reason behind this is the lack of well-defined metrics and testbed applications. Very often, metrics are too tightly related to a specific solution and thus cannot be reused for other systems or for comparisons. The lack of common metrics is also related to the lack of well-defined testbed applications. As said, foraging and construction are the only commonly used testbed applications. However, foraging is limited in its use to some collective behaviors, such as task allocation or area coverage and cannot be used in others. Moreover, as discussed in the taxonomy presented by Winfield (2009), there is no single definition of a standard foraging scenario. In order to promote the comparison of swarm robotics systems, it would be necessary to define a set of standard foraging scenarios and promote the distribution of open-source behaviors and public available datasets. Construction, as a testbed, suffers from similar limitations.

Operation and maintenance Swarm robotics systems have the potential advantage, over more traditional approaches, to require less manual intervention because of their robustness, scalability and flexibility. Although these three characteristics might reduce the need for maintenance, this might be true only up to a given extent. Further studies are necessary to understand when and how to perform maintenance on a swarm robotics system. Moreover, it is necessary to study if it is possible to derive general maintenance principles or if different collective behaviors need different maintenance approaches.

Regarding operation, one key issue is how to let humans and swarms cooperate. In fact, due to the lack of a centralized controller, it is in general very difficult to effectively control a swarm once it starts operating. This means that, for example, it might be difficult to stop a swarm that is behaving in an unpredicted or dangerous way. Some studies on human-swarm interaction have been recently published (see Sect. 3.4.3), but this issue still remains open.

In this paper, we presented a review of the state of the art in swarm robotics from a swarm engineering perspective. We foresee that, as swarm robotics is further developed and as it is used to tackle real-world applications, the need for a swarm engineering will increase. The 
goal of this paper was not only to present the significant works on methods and collective behaviors in swarm robotics, but also to propose a systematic categorization of their aspects. We think that such categorization effort is a necessary step for the development of a swarm engineering. In the conclusions, we also identified some open problems and underdeveloped aspects of swarm engineering.

Acknowledgements We thank the editor Lynne E. Parker and the anonymous reviewers for their feedback that helped improving the paper. We also thank the authors of the images reproduced in this paper for granting us publication permissions.

The research leading to the results presented in this paper has received funding from the European Research Council under the European Union's Seventh Framework Programme (FP7/2007-2013) / ERC grant agreement n 246939.

Manuele Brambilla, Mauro Birattari and Marco Dorigo acknowledge support from the F.R.S.-FNRS of Belgium's Wallonia-Brussels Federation, of which they are a F.R.I.A. Research Fellow, a Research Associate and a Research Director, respectively.

\section{References}

Abbott, R. (2006). Emergence explained. Complexity, 12(1), 13-26.

Agassounon, W., \& Martinoli, A. (2002). Efficiency and robustness of threshold-based distributed allocation algorithms in multi-agent systems. In Proceedings of the first international joint conference on autonomous agents and multi-agent systems (pp. 1090-1097). Richland: IFAAMAS.

Amé, J., Halloy, J., Rivault, C., Detrain, C., \& Deneubourg, J. L. (2006). Collegial decision making based on social amplification leads to optimal group formation. Proceedings of the National Academy of Sciences, 103(15), 5835-5840.

Ampatzis, C. (2008). On the evolution of autonomous time-based decision-making and communication in collective robotics. PhD thesis, IRIDIA, Université Libre de Bruxelles, Belgium.

Ampatzis, C., Tuci, E., Trianni, V., \& Dorigo, M. (2008). Evolution of signaling in a multi-robot system: categorization and communication. Adaptive Behavior, 16(1), 5-26.

Ampatzis, C., Tuci, E., Trianni, V., Christensen, A. L., \& Dorigo, M. (2009). Evolving self-assembly in autonomous homogeneous robots: experiments with two physical robots. Artificial Life, 15, 465-484.

Anderson, C., Theraulaz, G., \& Deneubourg, J.-L. (2002). Self-assemblages in insect societies. Insectes Sociaux, 49(2), 99-110.

Bachrach, J., Beal, J., \& McLurkin, J. (2010). Composable continuous-space programs for robotic swarms. Neural Computing \& Applications, 19(6), 825-847.

Bahçeci, E., \& Şahin, E. (2005). Evolving aggregation behaviors for swarm robotic systems: a systematic case study. In Proceedings of the 2005 swarm intelligence symposium, SIS 2005 (pp. 333-340). Piscataway: IEEE Press.

Bahçeci, E., Soysal, O., \& Şahin, E. (2003). A review: pattern formation and adaptation in multi-robot systems (Technical Report CMU-RI-TR-03-43). Robotics Institute, Carnegie Mellon University, Pittsburgh, PA.

Balch, T., \& Hybinette, M. (2000). Social potentials for scalable multi-robot formations. In Proceedings of the 2000 IEEE international conference on robotics and automation, ICRA 2000 (pp. 73-80). Piscataway: IEEE Press.

Baldassarre, G. (2006). Evolution of collective behaviour: coordination object retrieval in groups of physically-linked simulated robots. URL http://laral.istc.cnr.it/baldassarre/demos/2003swarmobject/ swarmobject.htm. Last checked on November 2012.

Baldassarre, G., Nolfi, S., \& Parisi, D. (2003). Evolving mobile robots able to display collective behaviors. Artificial Life, 9(3), 255-267.

Baldassarre, G., Parisi, D., \& Nolfi, S. (2006). Distributed coordination of simulated robots based on selforganization. Artificial Life, 12(3), 289-311.

Baldassarre, G., Trianni, V., Bonani, M., Mondada, F., Dorigo, M., \& Nolfi, S. (2007). Self-organized coordinated motion in groups of physically connected robots. IEEE Transactions on Systems, Man, and Cybernetics. Part B, 37(1), 224-239.

Bayindir, L., \& Şahin, E. (2007). A review of studies in swarm robotics. Turkish Journal of Electrical Engineering, 15(2), 115-147.

Beal, J. (2004). Programming an amorphous computational medium. In Lecture notes in computer science: Vol. 3566. Proceedings of the international workshop on unconventional programming paradigms $(U P P)$ (p. 97). Berlin: Springer. 
Beckers, R., Holland, O., \& Deneubourg, J.-L. (1994). From local actions to global tasks: stigmergy and collective robotics. In Artificial life IV (pp. 181-189). Cambridge: MIT Press.

Beer, R. D., \& Gallagher, J. C. (1992). Evolving dynamic neural networks for adaptive behavior. Adaptive Behavior, 1(1), 91-122.

Beni, G. (2005). From swarm intelligence to swarm robotics. In Lecture notes in computer science: Vol. 3342. Swarm robotics (pp. 1-9). Berlin: Springer.

Berman, S., Halász, Á. M., Hsieh, M. A., \& Kumar, V. (2009). Optimized stochastic policies for task allocation in swarms of robots. IEEE Transactions on Robotics, 25(4), 927-937.

Berman, S., Lindsey, Q., Sakar, M., Kumar, V., \& Pratt, S. (2011a). Experimental study and modeling of group retrieval in ants as an approach to collective transport in swarm robotic systems. Proceedings of the IEEE, 99(9), 1470-1481.

Berman, S., Nagpal, R., \& Halasz, A. (2011b). Optimization of stochastic strategies for spatially inhomogeneous robot swarms: a case study in commercial pollination. In IEEE/RSJ international conference on intelligent robots and systems (IROS) (pp. 3923-3930).

Bonabeau, E., Sobkowski, A., Theraulaz, G., \& Deneubourg, J.-L. (1997). Adaptive task allocation inspired by a model of division of labor in social insects. In Biocomputing and emergent computation: proceedings of BCEC97, London, UK (pp. 36-45). Singapore: World Scientific.

Bonabeau, E., Dorigo, M., \& Theraulaz, G. (1999). Swarm intelligence: from natural to artificial systems. New York: Oxford University Press.

Brambilla, M., Pinciroli, C., Birattari, M., \& Dorigo, M. (2009). A reliable distributed algorithm for group size estimation with minimal communication requirements. In Fourteenth international conference on advanced robotics-ICAR 2009 (p. 6). Proceedings on CD-ROM, paper ID 137.

Brambilla, M., Pinciroli, C., Birattari, M., \& Dorigo, M. (2012). Property-driven design for swarm robotics. In Proceedings of 11th international conference on autonomous agents and multiagent systems (AAMAS 2012) (pp. 139-146). Richland: IFAAMAS.

Breder, C. M. Jr. (1954). Equations descriptive of fish schools and other animal aggregations. Ecology, 35(3), 361-370.

Brooks, R. (1990). Elephants don't play chess. Robotics and Autonomous Systems, 6(1-2), 3-15.

Brooks, R. A. (1986). A robust layered control system for a mobile robot. IEEE Journal of Robotics and Automation, 2(1), 14-23.

Brutschy, A., Pini, G., \& Decugnière, A. (2012). Grippable objects for the foot-bot (Technical Report TR/IRIDIA/2012-001). IRIDIA, Université Libre de Bruxelles, Brussels, Belgium.

Camazine, S., Deneubourg, J.-L., Franks, N. R., Sneyd, J., Theraulaz, G., \& Bonabeau, E. (2001). Selforganization in biological systems. Princeton studies in complexity. Princeton: Princeton University Press.

Campo, A., \& Dorigo, M. (2007). Efficient multi-foraging in swarm robotics. In Lecture notes in artificial intelligence: Vol. 4648. Advances in artificial life, proceedings of ECAL 2007 (pp. 696-705). Berlin: Springer.

Campo, A., Nouyan, S., Birattari, M., Groß, R., \& Dorigo, M. (2006). Enhancing cooperative transport using negotiation of goal direction. In Lecture notes in computer science: Vol. 4150. Proceedings of the fifth international workshop on ant colony optimization and swarm intelligence (ANTS 2006) (pp. 365-366). Berlin: Springer.

Campo, A., Garnier, S., Dédriche, O., Zekkri, M., \& Dorigo, M. (2011). Self-organized discrimination of resources. PLoS ONE, 6(5), 05.

Cao, Y. U., Fukunaga, A. S., Kahng, A. B., \& Meng, F. (1997). Cooperative mobile robotics: antecedents and directions. Autonomous Robots, 4(1), 7-27.

Çelikkanat, H., \& Şahin, E. (2010). Steering self-organized robot flocks through externally guided individuals. Neural Computing \& Applications, 19(6), 849-865.

Christensen, A. L., O’Grady, R., \& Dorigo, M. (2008). SWARMORPH-script: a language for arbitrary morphology generation in self-assembling robots. Swarm Intelligence, 2(2-4), 143-165.

Christensen, A. L., O'Grady, R., \& Dorigo, M. (2009). From fireflies to fault-tolerant swarms of robots. IEEE Transactions on Evolutionary Computation, 13(4), 754-766.

Correll, N. (2008). Parameter estimation and optimal control of swarm-robotic systems: a case study in distributed task allocation. In IEEE international conference on robotics and automation (ICRA) (pp. 33023307).

Correll, N., \& Martinoli, A. (2007). Modeling self-organized aggregation in a swarm of miniature robots. In IEEE international conference on robotics and automation.

Couzin, I. D., Krause, J., Franks, N. R., \& Levin, S. A. (2005). Effective leadership and decision-making in animal groups on the move. Nature, 433(7025), 513-516.

Crespi, V., Galstyan, A., \& Lerman, K. (2008). Top-down vs bottom-up methodologies in multi-agent system design. Autonomous Robots, 24(3), 303-313. 
Dantu, K., Berman, S., Kate, B., \& Nagpal, R. (2012). A comparison of deterministic and stochastic approaches for allocating spatially dependent tasks in micro-aerial vehicle collectives. In IEEE/RSJ international conference on intelligent robots and systems.

Deneubourg, J.-L., Aron, S., Goss, S., \& Pasteels, J. M. (1990). The self-organizing exploratory pattern of the argentine ant. Journal of Insect Behavior, 3(2), 159-168.

Di Caro, G. A., Ducatelle, F., \& Gambardella, L. M. (2009). Wireless communications for distributed navigation in robot swarms. In Lecture notes in computer science: Vol. 5484. Applications of evolutionary computing (pp. 21-30). Berlin: Springer.

Dixon, C., Winfield, A., \& Fisher, M. (2011). Towards temporal verification of emergent behaviours in swarm robotic systems. In Lecture notes in computer science: Vol. 6856. Towards autonomous robotic systems (pp. 336-347). Berlin: Springer.

Donald, B. R., Jennings, J., \& Rus, D. (1997). Information invariants for distributed manipulation. The International Journal of Robotics Research, 16(5), 673-702.

Dorigo, M., \& Birattari, M. (2007). Swarm intelligence. Scholarpedia, 2(9), 1462.

Dorigo, M., \& Şahin, E. (2004). Guest editorial. Autonomous Robots, 17, 111-113.

Dorigo, M., Tuci, E., Trianni, V., Groß, R., Nouyan, S., Ampatzis, C., Labella, T. H., O’Grady, R., Bonani, M., \& Mondada, F. (2006). SWARM-BOT: design and implementation of colonies of self-assembling robots. In Computational intelligence: principles and practice (pp. 103-135). New York: IEEE Computational Intelligence Society. Chap. 6.

Dorigo, M., Floreano, D., Gambardella, L., Mondada, F., Nolfi, S., Baaboura, T., Birattari, M., Bonani, M., Brambilla, M., Brutschy, A., Burnier, D., Campo, A., Christensen, A., Decugnière, A., Di Caro, G., Ducatelle, F., Ferrante, E., Forster, A., Martinez Gonzales, J., Guzzi, J., Longchamp, V., Magnenat, S., Mathews, N., Montes de Oca, M., O’Grady, R., Pinciroli, C., Pini, G., Retornaz, P., Roberts, J., Sperati, V., Stirling, T., Stranieri, A., Stutzle, T., Trianni, V., Tuci, E., Turgut, A., \& Vaussard, F. (2012). Swarmanoid: a novel concept for the study of heterogeneous robotic swarms. IEEE Robotics \& Automation Magazine, in press.

Ducatelle, F., Di Caro, G. A., Pinciroli, C., Mondada, F., \& Gambardella, L. M. (2011a). Communication assisted navigation in robotic swarms: self-organization and cooperation. In Proceedings of the IEEE/RSJ international conference on intelligent robots and systems (IROS 2011) (pp. 4981-4988). Los Alamitos: IEEE Computer Society Press.

Ducatelle, F., Di Caro, C. P. G. A., \& Gambardella, L. M. (2011b). Self-organized cooperation between robotic swarms. Swarm Intelligence, 5(2), 73-96.

Dudek, G., Jenkin, M., Milios, E., \& Wilkes, D. (1993). A taxonomy for swarm robots. In Proceedings of the 1993 IEEE/RSJ international conference on intelligent robots and systems, IROS 93 (pp. 441-447). Piscataway: IEEE Press.

Elman, J. L. (1990). Finding structure in time. Cognitive Science, 14(2), 179-211.

Ferrante, E., Turgut, A. E., Mathews, N., Birattari, M., \& Dorigo, M. (2010). Flocking in stationary and non-stationary environments: a novel communication strategy for heading alignment. In Lecture notes in computer science: Vol. 6239. Parallel problem solving from nature-PPSN XI: 11th international conference (pp. 331-340). Berlin: Springer.

Ferrante, E., Turgut, A. E., Huepe, C., Stranieri, A., Pinciroli, C., \& Dorigo, M. (2012). Self-organized flocking with a mobile robot swarm: a novel motion control method. Adaptive Behavior.

Ferrante, E., Brambilla, M., Birattari, M., \& Dorigo, M. (2013). Socially-mediated negotiation for obstacle avoidance in collective transport. In Springer tracts in advanced robotics: Vol. 83. Proceedings of the international symposium on distributed autonomous robotics systems (DARS 2010) (pp. 571-583). Berlin: Springer.

Fine, T. L. (1999). Feedforward neural network methodology. Berlin: Springer.

Flocchini, P., Prencipe, G., Santoro, N., \& Widmayer, P. (2008). Arbitrary pattern formation by asynchronous, anonymous, oblivious robots. Theoretical Computer Science, 407(1-3), 412-447.

Francesca, G., Brambilla, M., Trianni, V., Dorigo, M., \& Birattari, M. (2012). Analysing an evolved robotic behaviour using a biological model of collegial decision making. In Lecture notes in computer science: Vol. 7426. Proceedings of the 12th international conference on adaptive behavior (SAB2012) (pp. 381390). Berlin: Springer.

Franks, N., \& Sendova-Franks, A. (1992). Brood sorting by ants: distributing the workload over the worksurface. Behavioral Ecology and Sociobiology, 30, 109-123.

Friedmann, M. (2010). Simulation of autonomous robot teams with adaptable level of abstraction. Ph.D. thesis, University of Darmstadt, Germany.

Frigg, R., \& Hartmann, S. (2012). Models in science. In The Stanford encyclopedia of philosophy. Stanford: Stanford University. Spring 2012 edition.

Galstyan, A., Hogg, T., \& Lerman, K. (2005). Modeling and mathematical analysis of swarms of microscopic robots. In Proceedings of the 2005 swarm intelligence symposium-(SIS 2005) (pp. 201-208). Los Alamitos: IEEE Computer Society Press. 
Garnier, S., Jost, C., Jeanson, R., Gautrais, J., Asadpour, M., Caprari, G., \& Theraulaz, G. (2005). Aggregation behaviour as a source of collective decision in a group of cockroach-like robots. In Lecture notes in artificial intelligence: Vol. 3630. Advances in artificial life (pp. 169-178). Berlin: Springer.

Garnier, S., Gautrais, J., Asadpour, M., Jost, C., \& Theraulaz, G. (2009). Self-organized aggregation triggers collective decision making in a group of cockroach-like robots. Adaptive Behavior, 17(2), 109-133.

Gazi, V., \& Fidan, B. (2007). Coordination and control of multi-agent dynamic systems: models and approaches. In Lecture notes in computer science: Vol. 4433. Swarm robotics (pp. 71-102). Berlin: Springer.

Gazi, V., \& Passino, K. M. (2002). Stability analysis of social foraging swarms: combined effects of attractant/repellent profiles. In Proceedings of the 41st IEEE conference on decision and control (Vol. 3, pp. 2848-2853). Piscataway: IEEE Press.

Gazi, V., \& Passino, K. M. (2003). Stability analysis of swarms. IEEE Transactions on Automatic Control, 48(4), 692-696.

Gazi, V., \& Passino, K. M. (2004a). A class of attractions/repulsion functions for stable swarm aggregations. International Journal of Control, 77(18), 1567-1579.

Gazi, V., \& Passino, K. M. (2004b). Stability analysis of social foraging swarms. IEEE Transactions on Systems, Man, and Cybernetics. Part B, 34(1), 539-557.

Gazi, V., \& Passino, K. M. (2005). Stability of a one-dimensional discrete-time asynchronous swarm. IEEE Transactions on Systems, Man, and Cybernetics. Part B, 35(4), 834-841.

Getling, A. V. (1998). Rayleigh-Bénard convection: structures and dynamics (Vol. 11). London: World Scientific.

Giusti, A., Nagi, J., Gambardella, L., \& Caro, G. D. (2012). Distributed consensus for interaction between humans and mobile robot swarms. In Proceedings of 11th international conference on autonomous agents and multiagent systems (AAMAS 2012), Richland, SC.

Goldberg, D. E. (1989). Genetic algorithms in search, optimization, and machine learning. Reading: Addison-Wesley.

Granovetter, M. (1978). Threshold models of collective behavior. American Journal of Sociology, 83(6), $1420-1443$.

Grassé, P.-P. (1959). La reconstruction du nid et les coordinations interindividuelles chez bellicositermes natalensis et cubitermes sp. la théorie de la stigmergie: Essai d'interprétation du comportement des termites constructeurs. Insectes Sociaux, 6, 41-80.

Groß, R., \& Dorigo, M. (2008a). Evolution of solitary and group transport behaviors for autonomous robots capable of self-assembling. Adaptive Behavior, 16(5), 285-305.

Groß, R., \& Dorigo, M. (2008b). Self-assembly at the macroscopic scale. Proceedings of the IEEE, 96(9), 1490-1508.

Groß, R., \& Dorigo, M. (2009). Towards group transport by swarms of robots. International Journal of BioInspired Computation, 1(1-2), 1-13.

Grünbaum, D., \& Okubo, A. (1994). Modeling social animal aggregations. Frontiers in Theoretical Biology, $100,296-325$.

Gutiérrez, Á., Campo, A., Monasterio-Huelin, F., Magdalena, L., \& Dorigo, M. (2010). Collective decisionmaking based on social odometry. Neural Computing \& Applications, 19(6), 807-823.

Halász, A., Liang, Y., Hsieh, M., \& Lai, H.-J. (2012). Emergence of specialization in a swarm of robots. In Springer tracts in advanced robotics: Vol. 83. Distributed autonomous robotic systems (pp. 403-416). Berlin: Springer.

Hamann, H. (2012). Towards swarm calculus: universal properties of swarm performance and collective decisions. In Lecture notes in computer science: Vol. 7461. Swarm intelligence: 8th international conference, ANTS 2012 (pp. 168-179). Berlin: Springer.

Hamann, H., \& Wörn, H. (2008). A framework of space-time continuous models for algorithm design in swarm robotics. Swarm Intelligence, 2(2-4), 209-239.

Hettiarachchi, S. D. (2007). Distributed evolution for swarm robotics. PhD thesis, University of Wyoming, Laramie, WY.

Holland, J. H. (1975). Adaptation in natural and artificial systems: an introductory analysis with applications to biology, control, and artificial intelligence. Cambridge: MIT Press.

Howard, A., Matarić, M. J., \& Sukhatme, G. S. (2002). Mobile sensor network deployment using potential fields: a distributed, scalable solution to the area coverage problem. In Proceedings of the 2002 international symposium on distributed autonomous robotic systems (DARS 2002) (pp. 299-308). Berlin: Springer.

Hsieh, M. A., Halász, Á., Berman, S., \& Kumar, V. (2008). Biologically inspired redistribution of a swarm of robots among multiple sites. Swarm Intelligence, 2(2-4), 121-141.

Iocchi, L., Nardi, D., \& Salerno, M. (2001). Reactivity and deliberation: a survey on multi-robot systems. In Lecture notes in computer science: Vol. 2103. Balancing reactivity and social deliberation in multi-agent systems (pp. 9-32). Berlin: Springer. 
Jeanson, R., Rivault, C., Deneubourg, J.-L., Blanco, S., Fournier, R., Jost, C., \& Theraulaz, G. (2005). Selforganized aggregation in cockroaches. Animal Behaviour, 69(1), 169-180.

Kaelbling, L. P., Littman, M. L., \& Moore, A. W. (1996). Reinforcement learning: a survey. Journal of Artificial Intelligence Research, 4, 237-285.

Kaelbling, L. P., Littman, M. L., \& Cassandra, A. R. (1998). Planning and acting in partially observable stochastic domains. Artificial Intelligence, 101(1-2), 99-134.

Kalyanakrishnan, S., \& Stone, P. (2007). Batch reinforcement learning in a complex domain. In AAMAS '07: proceedings of the 6th international joint conference on autonomous agents and multiagent systems. Richland: IFAAMAS.

Kaminka, G. A., Schechter-Glick, R., \& Sadov, V. (2008). Using sensor morphology for multirobot formations. IEEE Transactions on Robotics, 24(2), 271-282.

Kazadi, S. (2000). Swarm engineering. Ph.D. thesis, California Institute of Technology, Pasadena, CA, USA.

Kazadi, S. (2009). Model independence in swarm robotics. International Journal of Intelligent Computing and Cybernetics, 2(4), 672-694.

Kendall, D. G. (1966). Branching processes since 1873. Journal of the London Mathematical Society, 41(1), 386-406.

Khatib, O. (1986). Real-time obstacle avoidance for manipulators and mobile robots. The International Journal of Robotics Research, 5(1), 90-98.

Kolling, A., Nunnally, S., \& Lewis, M. (2012). Towards human control of robot swarms. In Proceedings of the seventh annual ACM/IEEE international conference on human-robot interaction (pp. 89-96). New York: ACM.

Konur, S., Dixon, C., \& Fisher, M. (2012). Analysing robot swarm behaviour via probabilistic model checking. Robotics and Autonomous Systems, 60(2), 199-213.

Kramer, J., \& Scheutz, M. (2007). Development environments for autonomous mobile robots: a survey. $A u$ tonomous Robots, 22(2), 101-132.

Krieger, M. J. B., \& Billeter, J.-B. (2000). The call of duty: self-organised task allocation in a population of up to twelve mobile robots. Robotics and Autonomous Systems, 30(1-2), 65-84.

Kube, C. R., \& Bonabeau, E. (2000). Cooperative transport by ants and robots. Robotics and Autonomous Systems, 30(1-2), 85-101.

Labella, T. H., Dorigo, M., \& Deneubourg, J.-L. (2006). Division of labour in a group of robots inspired by ants' foraging behaviour. ACM Transactions on Autonomous and Adaptive Systems, 1(1), 4-25.

Langer, J. S. (1980). Instabilities and pattern formation in crystal growth. Reviews of Modern Physics, 52(1), $1-28$.

Lee, J., \& Arkin, R. C. (2003). Adaptive multi-robot behavior via learning momentum. In IEEE international conference on intelligent robots and systems (IROS 2003) (Vol. 2). Piscataway: IEEE Press.

Lerman, K., \& Galstyan, A. (2002). Mathematical model of foraging in a group of robots: effect of interference. Autonomous Robots, 13(2), 127-141.

Lerman, K., Galstyan, A., Martinoli, A., \& Ijspeert, A. J. (2001). A macroscopic analytical model of collaboration in distributed robotic systems. Artificial Life, 7(4), 375-393.

Levi, P., \& Kernbach, S. (2010). Symbiotic multi-robot organisms. Berlin: Springer.

Li, L., Martinoli, A., \& Abu-Mostafa, Y. S. (2004). Learning and measuring specialization in collaborative swarm systems. Adaptive Behavior, 12(3-4), 199-212.

Lindsey, Q., Mellinger, D., \& Kumar, V. (2012). Construction with quadrotor teams. Autonomous Robots, 33, $323-336$.

Liu, W. (2007). Modelling of adaptive foraging in swarm robotic systems. URL http://www.brl.ac.uk/ researchthemes/swarmrobotics/swarmroboticsystems.aspx. Last checked on November 2012.

Liu, W., \& Winfield, A. (2010). Modeling and optimization of adaptive foraging in swarm robotic systems. International Journal of Robotics Research, 29(14), 1743-1760.

Liu, W., Winfield, A. F. T., Sa, J., Chen, J., \& Dou, L. (2007). Towards energy optimization: emergent task allocation in a swarm of foraging robots. Adaptive Behavior, 15(3), 289-305.

Liu, Y., \& Passino, K. M. (2004). Stable social foraging swarms in a noisy environment. IEEE Transactions on Automatic Control, 49(1), 30-44.

Liu, Y., Passino, K. M., \& Polycarpou, M. M. (2003). Stability analysis of m-dimensional asynchronous swarms with a fixed communication topology. IEEE Transactions on Automatic Control, 48(1), 76-95.

Martinoli, A., Ijspeert, A. J., \& Mondada, F. (1999). Understanding collective aggregation mechanisms: from probabilistic modelling to experiments with real robots. Robotics and Autonomous Systems, 29(1), 5163.

Martinoli, A., Easton, K., \& Agassounon, W. (2004). Modeling swarm robotic systems: a case study in collaborative distributed manipulation. The International Journal of Robotics Research, 23(4-5), 415-436.

Massink, M., Brambilla, M., Latella, D., Dorigo, M., \& Birattari, M. (2012). Analysing robot swarm decisionmaking with bio-pepa. In Lecture notes in computer science: Vol. 7461. Swarm intelligence (pp. 25-36). Berlin: Springer. 
Matarić, M. J. (1997). Reinforcement learning in the multi-robot domain. Autonomous Robots, 4(1), 73-83.

Matarić, M. J. (1998). Using communication to reduce locality in distributed multi-agent learning. Journal of Experimental and Theoretical Artificial Intelligence, 10(3), 357-369.

Matarić, M. J., \& Cliff, D. (1996). Challenges in evolving controllers for physical robots. Robotics and Autonomous Systems, 19(1), 67-83.

Mathews, N., Christensen, A. L., Ferrante, E., O’Grady, R., \& Dorigo, M. (2010). Establishing spatially targeted communication in a heterogeneous robot swarm. In Proceedings of 9 th international conference on autonomous agents and multiagent systems (AAMAS 2010) (pp. 939-946). Richland: IFAAMAS.

Mathews, N., Christensen, A. L., O'Grady, R., \& Dorigo, M. (2012). Spatially targeted communication and self-assembly. In Proceedings of the 2012 IEEE/RSJ international conference on intelligent robots and systems (IROS 2012) (pp. 2678-2679). Los Alamitos: IEEE Computer Society Press.

Maxim, P. M., Spears, W. M., \& Spears, D. F. (2009). Robotic chain formations. In Proceedings of the IFAC workshop on networked robotics (pp. 19-24). Oxford: Elsevier.

McLurkin, J., Smith, J., Frankel, J., Sotkowitz, D., Blau, D., \& Schmidt, B. (2006). Speaking swarmish: human-robot interface design for large swarms of autonomous mobile robots. In 2006 AAAI spring symposium (pp. 72-75). Menlo Park: AAAI.

Meinhardt, H. (1982). Models of biological pattern formation (Vol. 6). London: Academic Press.

Melhuish, C. (1999). Intelligent Autonomous Systems Laboratory. URL http://www.ias.uwe.ac.uk/. Last checked on November 2012.

Melhuish, C., Holland, O., \& Hoddell, S. (1999a). Convoying: using chorusing for the formation of travelling groups of minimal agents. Robotics and Autonomous Systems, 28(2-3), 207-216.

Melhuish, C., Welsby, J., \& Edwards, C. (1999b). Using templates for defensive wall building with autonomous mobile ant-like robots. In Proceedings of towards intelligent and autonomous mobile robots (Vol. 99).

Minsky, M. (1967). Computation: finite and infinite machines. Upper Saddle River: Prentice-Hall.

Mondada, F. (2005). Swarm-bots. URL http://www.swarm-bot.org/. Last checked on November 2012.

Mondada, F., Bonani, M., Guignard, A., Magnenat, S., Studer, C., \& Floreano, D. (2005). Superlinear physical performances in a SWARM-BOT. In Lecture notes in computer science: Vol. 3630. Proceedings of the VIIIth European conference on artificial life (pp. 282-291). Berlin: Springer.

Montes de Oca, M. A., Ferrante, E., Scheidler, A., Pinciroli, C., Birattari, M., \& Dorigo, M. (2011). Majorityrule opinion dynamics with differential latency: a mechanism for self-organized collective decisionmaking. Swarm Intelligence, 5(3-4), 305-327.

Naghsh, A., Gancet, J., Tanoto, A., \& Roast, C. (2008). Analysis and design of human-robot swarm interaction in firefighting. In Proceedings of the 17th IEEE international symposium on the robot and human interactive communication (Ro-man 2008) (pp. 255-260).

Nolfi, S., \& Floreano, D. (2000). Evolutionary robotics. intelligent robots and autonomous agents. Cambridge: MIT Press.

Nouyan, S., Campo, A., \& Dorigo, M. (2008). Path formation in a robot swarm: self-organized strategies to find your way home. Swarm Intelligence, 2(1), 1-23.

Nouyan, S., Groß, R., Bonani, M., Mondada, F., \& Dorigo, M. (2009). Teamwork in self-organized robot colonies. IEEE Transactions on Evolutionary Computation, 13(4), 695-711.

O’Grady, R., Christensen, A., \& Dorigo, M. (2009a). SWARMORPH: multi-robot morphogenesis using directional self-assembly. IEEE Transactions on Robotics, 25(3), 738-743.

O’Grady, R., Pinciroli, C., Christensen, A. L., \& Dorigo, M. (2009b). Supervised group size regulation in a heterogeneous robotic swarm. In 9th conference on autonomous robot systems and competitions, robótica 2009 (pp. 113-119). Castelo Branco: IPCB-Instituto Politécnico de Castelo Branco.

O’Grady, R., Groß, R., Christensen, A. L., \& Dorigo, M. (2010). Self-assembly strategies in a group of autonomous mobile robots. Autonomous Robots, 28(4), 439-455.

O’Hara, K. J., \& Balch, T. (2007). Pervasive sensor-less networks for cooperative multi-robot tasks. In Distributed autonomous robotic systems 6 (pp. 305-314). Tokyo: Springer.

Okubo, A. (1986). Dynamical aspects of animal grouping: swarms, schools, flocks, and herds. Advances in Biophysics, 22(0), 1-94.

Panait, L., \& Luke, S. (2005). Cooperative multi-agent learning: the state of the art. Autonomous Agents and Multi-Agent Systems, 11(3), 387-434.

Parker, C. A. C., \& Zhang, H. (2011). Biologically inspired collective comparisons by robotic swarms. International Journal of Robotics Research, 30(5), 524-535.

Parker, L. E. (1996). L-ALLIANCE: task-oriented multi-robot learning in behavior-based systems. Advanced Robotics, 11(4), 305-322.

Parrish, J. K., Viscido, S. V., \& Grünbaum, D. (2002). Self-organized fish schools: an examination of emergent properties. Biological Bulletin, 202(3), 296-305. 
Payton, D., Daily, M., Estowski, R., Howard, M., \& Lee, C. (2001). Pheromone robotics. Autonomous Robots, 11(3), 319-324.

Pinciroli, C., O’Grady, R., Christensen, A. L., \& Dorigo, M. (2009). Self-organised recruitment in a heterogeneous swarm. In Fourteenth international conference on advanced robotics-ICAR 2009 (p. 6). Proceedings on CD-ROM, paper ID 176.

Pinciroli, C., O’Grady, R., Christensen, A. L., \& Dorigo, M. (2010). Heterogeneous swarms through minimal communication between homogeneous sub-swarms. In Lecture notes in computer science: Vol. 6234. Proceedings of the seventh international conference on ant colony optimization and swarm intelligence (ANTS-2010) (pp. 558-559). Berlin: Springer.

Pinciroli, C., Trianni, V., O’Grady, R., Pini, G., Brutschy, A., Brambilla, M., Mathews, N., Ferrante, E., Di Caro, G., Ducatelle, F., Birattari, M., Gambardella, L. M., \& Dorigo, M. (2012). ARGoS: a modular, parallel, multi-engine simulator for multi-robot systems. Swarm Intelligence, 6(4).

Pini, G. (2011). Task partitioning in swarms of robots an adaptive method for strategy selection. URL http://iridia.ulb.ac.be/supp/IridiaSupp2011-003/index.html. Last checked on November 2012.

Pini, G., \& Tuci, E. (2008). On the design of neuro-controllers for individual and social learning behaviour in autonomous robots: an evolutionary approach. Connection Science, 20(2-3), 211-230.

Pini, G., Brutschy, A., Birattari, M., \& Dorigo, M. (2009). Interference reduction through task partitioning in a robotic swarm. In IEEE international conference on neural networks: IEEE world congress on computational intelligence. Setubal: INSTICC Press.

Pini, G., Brutschy, A., Frison, M., Roli, A., Dorigo, M., \& Birattari, M. (2011). Task partitioning in swarms of robots: an adaptive method for strategy selection. Swarm Intelligence, 5(3-4), 283-304.

Podevijn, G., O'Grady, R., \& Dorigo, M. (2012). Self-organised feedback in human swarm interaction. In Proceedings of the workshop on robot feedback in human-robot interaction: how to make a robot readable for a human interaction partner (Ro-Man 2012).

Prorok, A., Correll, N., \& Martinoli, A. (2011). Multi-level spatial modeling for stochastic distributed robotic systems. The International Journal of Robotics Research, 30(5), 574-589.

Pugh, J., \& Martinoli, A. (2007). Parallel learning in heterogeneous multi-robot swarms. In Proceedings of the IEEE congress on evolutionary computation (pp. 3839-3846). Piscataway: IEEE Press.

Reif, J. H., \& Wang, J. (1999). Social potential fields: a distributed behavioral control for autonomous robots. Robotics and Autonomous Systems, 27(3), 171-194.

Reynolds, C. (1987a). Boids (Flocks, herds, and schools: a distributed behavioral model). URL http://www.red3d.com/cwr/boids/. Last checked on November 2012.

Reynolds, C. W. (1987b). Flocks, herds and schools: a distributed behavioral model. Computer Graphics, 21(4), 25-34.

Riedmiller, M., Gabel, T., Hafner, R., \& Lange, S. (2009). Reinforcement learning for robot soccer. $A u$ tonomous Robots, 27(1), 55-73.

Rosenfeld, A., Kaminka, G. A., Kraus, S., \& Shehory, O. (2008). A study of mechanisms for improving robotic group performance. Artificial Intelligence, 172(6-7), 633-655.

Şahin, E. (2005). Swarm robotics: from sources of inspiration to domains of application. In Lecture notes in computer science: Vol. 3342. Swarm robotics (pp. 10-20). Berlin: Springer.

Scheidler, A. (2011). Dynamics of majority rule with differential latencies. Physical Review E, 83(3), 031116.

Schmickl, T., Hamann, H., Wörn, H., \& Crailsheim, K. (2009). Two different approaches to a macroscopic model of a bio-inspired robotic swarm. Robotics and Autonomous Systems, 57(9), 913-921.

Schwager, M., Michael, N., Kumar, V., \& Rus, D. (2011). Time scales and stability in networked multi-robot systems. In Proceedings of the IEEE international conference on robotics and automation (ICRA) (pp. 3855-3862).

Shucker, B., \& Bennett, J. K. (2007). Scalable control of distributed robotic macrosensors. In Distributed autonomous robotic systems 6 (pp. 379-388). Tokyo: Springer.

Shucker, B., Murphey, T., \& Bennett, J. (2008). Convergence-preserving switching for topology-dependent decentralized systems. IEEE Transactions on Robotics, 24(6), 1405-1415.

Soysal, O., \& Şahin, E. (2005). Probabilistic aggregation strategies in swarm robotic systems. In Proceedings of the IEEE swarm intelligence symposium (pp. 325-332). Piscataway: IEEE Press.

Soysal, O., \& Şahin, E. (2007). A macroscopic model for self-organized aggregation in swarm robotic systems. In Lecture notes in computer science: Vol. 4433. Swarm robotics (pp. 27-42). Berlin: Springer.

Soysal, O., Bahçeci, E., \& Şahin, E. (2007). Aggregation in swarm robotic systems: evolution and probabilistic control. Turkish Journal of Electrical Engineering and Computer Sciences, 15(2), 199-225.

Spears, W. M., \& Spears, D. F. (2012). Physics-based swarm intelligence. Berlin: Springer.

Spears, W. M., Spears, D. F., Hamann, J. C., \& Heil, R. (2004). Distributed, physics-based control of swarms of vehicles. Autonomous Robots, 17(2-3), 137-162.

Sperati, V., Trianni, V., \& Nolfi, S. (2008). Evolving coordinated group behaviours through maximization of mean mutual information. Swarm Intelligence, 2(2-4), 73-95. 
Sperati, V., Trianni, V., \& Nolfi, S. (2011). Self-organised path formation in a swarm of robots. Swarm Intelligence, 5, 97-119.

Stewart, R. L., \& Russell, R. A. (2006). A distributed feedback mechanism to regulate wall construction by a robotic swarm. Adaptive Behavior, 14, 21-51.

Stirling, T., \& Floreano, D. (2010). Energy efficient swarm deployment for search in unknown environments. In Lecture notes in computer science. Proceedings of the 7th international conference on swarm intelligence (ANTS 2010) (pp. 562-563). Berlin: Springer.

Stone, P., \& Veloso, M. M. (2000). Multiagent systems: a survey from a machine learning perspective. $A u$ tonomous Robots, 8(3), 345-383.

Stranieri, A., Ferrante, E., Turgut, A. E., Trianni, V., Pinciroli, C., Birattari, M., \& Dorigo, M. (2011). Selforganized flocking with a heterogeneous mobile robot swarm. In Advances in artificial life, ECAL 2011 (pp. 789-796). Cambridge: MIT Press.

Sutton, R. S., \& Barto, A. G. (1998). Reinforcement learning: an introduction. Cambridge: MIT Press.

Theraulaz, G., Goss, S., Gervet, J., \& Deneubourg, J.-L. (1990). Task differentiation in polistes wasp colonies: a model for self-organizing groups of robots. In Proceedings of the first international conference on simulation of adaptive behavior on from animals to animats (pp. 346-355). Cambridge: MIT Press.

Theraulaz, G., Bonabeau, E., \& Deneubourg, J.-L. (1998). Response threshold reinforcements and division of labour in insect societies. Proceedings of the Royal Society B. Biological Sciences, 265(1393), 327-332.

Trianni, V., \& Dorigo, M. (2006). Self-organisation and communication in groups of simulated and physical robots. Biological Cybernetics, 95, 213-231.

Trianni, V., Labella, T. H., Groß, R., Şahin, E., Dorigo, M., \& Deneubourg, J.-L. (2002). Modeling pattern formation in a swarm of self-assembling robots (Technical Report TR/IRIDIA/2002-12). IRIDIA, Université Libre de Bruxelles, Belgium.

Trianni, V., Groß, R., Labella, T. H., Şahin, E., \& Dorigo, M. (2003). Evolving aggregation behaviors in a swarm of robots. In Lecture notes in artificial intelligence: Vol. 2801. Advances in artificial life: 7 th European conference-ECAL 2003 (pp. 865-874). Berlin: Springer.

Tuci, E., Trianni, V., \& Dorigo, M. (2004). 'Feeling' the flow of time through sensorymotor coordination. Connection Science, 16(4), 301-324.

Turgut, A. E., Çelikkanat, H., Gökçe, F., \& Şahin, E. (2008a). Self-organized flocking in mobile robot swarms. Swarm Intelligence, 2(2-4), 97-120.

Turgut, A. E., Huepe, C., Çelikkanat, H., Gökçe, F., \& Şahin, E. (2008b). Modeling phase transition in selforganized mobile robot flocks. In Lecture notes in computer science: Vol. 5217. Proceedings of the 6th international conference on ant colony optimization and swarm intelligence, ANTS 2008 (pp. 108-119). Berlin: Springer.

Turing, A. (1953). The chemical basis of morphogenesis. Philosophical Transactions of the Royal Society. Part B, 237, 37-72.

Varghese, B., \& McKee, G. (2009). A review and implementation of swarm pattern formation and transformation models. International Journal of Intelligent Computing and Cybernetics, 2(4), 786-817.

Vaughan, R. T. (2008). Massively multi-robot simulation in stage. Swarm Intelligence, 2(2-4), 189-208.

Waibel, M., Keller, L., \& Floreano, D. (2009). Genetic team composition and level of selection in the evolution of cooperation. IEEE Transactions on Evolutionary Computation, 13(3), 648-660.

Wang, B., Lim, H. B., \& Ma, D. (2009). A survey of movement strategies for improving network coverage in wireless sensor networks. Computer Communications, 32(13-14), 1427-1436.

Wawerla, J., Sukhatme, G. S., \& Matarić, M. J. (2002). Collective construction with multiple robots. In Proceedings of the IEEE/RSJ international conference on intelligent robots and systems (IROS) (pp. 2696-2701).

Werfel, J. (2006). Extended stigmergy in collective construction. IEEE Intelligent Systems, 21, 20-28.

Werfel, J. (2011). Distributed multi-robot algorithms for the TERMES 3D collective construction system. URL http://www.eecs.harvard.edu/ssr/publications/. Last checked on November 2012.

Werfel, J., \& Nagpal, R. (2008). Three-dimensional construction with mobile robots and modular blocks. International Journal of Robotics Research, 27(3-4), 463-479.

Werfel, J., Petersen, K., \& Nagpal, R. (2011). Distributed multi-robot algorithms for the TERMES 3D collective construction system. In Proceedings of the IEEE/RSJ international conference on intelligent robots and systems (IROS).

Wessnitzer, J., \& Melhuish, C. (2003). Collective decision-making and behaviour transitions in distributed ad hoc wireless networks of mobile robots: target-hunting. In Lecture notes in computer science: Vol. 2801. Advances in artificial life (pp. 893-902). Berlin: Springer.

Winfield, A. F. T. (2009). Towards an engineering science of robot foraging. In Distributed autonomous robotic systems 8 (pp. 185-192). Berlin: Springer.

Winfield, A. F. T., Harper, C. J., \& Nembrini, J. (2004). Towards dependable swarms and a new discipline of swarm engineering. In Lecture notes in computer science: Vol. 3342. Proceedings of the international workshop on simulation of adaptive behavior, SAB 2004 (pp. 126-142). Berlin: Springer. 
Winfield, A. F. T., Sa, J., Fernandez-Gago, M. C., Dixon, C., \& Fisher, M. (2005). On formal specification of emergent behaviours in swarm robotic systems. International Journal of Advanced Robotic Systems, 2(4), 363-370.

Winfield, A. F. T., Liu, W., Nembrini, J., \& Martinoli, A. (2008). Modelling a wireless connected swarm of mobile robots. Swarm Intelligence, 2(2-4), 241-266.

Wolpert, D. H., \& Tumer, K. (1999). An introduction to collective intelligence (Technical Report NASAARC-IC-99-63). NASA Ames Research Center.

Yang, E., \& Gu, D. (2005). A survey on multiagent reinforcement learning towards multi-robot systems. In Proceedings of IEEE symposium on computational intelligence and games. Piscataway: IEEE Press.

Yun, S., Schwager, M., \& Rus, D. (2009). Coordinating construction of truss structures using distributed equal-mass partitioning. In Springer tracts in advanced robotics: Vol. 70. Proc. of the 14th international symposium on robotics research (pp. 607-623). Berlin: Springer. 